\title{
FIRST KECK NULLING OBSERVATIONS OF A YOUNG STELLAR OBJECT: PROBING THE CIRCUMSTELLAR ENVIRONMENT OF THE HERBIG Ae STAR MWC 325
}

\author{
S. Ragland ${ }^{1}$, K. Ohnaka ${ }^{2}$, L. Hillenbrand ${ }^{3}$, S. T. Ridgway ${ }^{4}$, M. M. Colavita ${ }^{5}$, R. L. Akeson ${ }^{6}$, \\ W. Cotton $^{7}$, W. C. Danchi ${ }^{8}$, M. Hrynevich ${ }^{1}$, R. Millan-Gabet ${ }^{6}$, and W. A. Traub ${ }^{5}$ \\ ${ }^{1}$ W. M. Keck Observatory, Kamuela, HI 96743, USA; sragland@keck.hawaii.edu \\ ${ }^{2}$ Max-Planck-Institut für Radioastronomie, 53121 Bonn, Germany \\ ${ }^{3}$ Astrophysics Department, California Institute of Technology, Pasadena, CA 91125, USA \\ ${ }^{4}$ National Optical Astronomy Observatories, Tucson, AZ 85726-6732, USA \\ 5 Jet Propulsion Laboratory, California Institute of Technology, Pasadena CA, 91109, USA \\ ${ }^{6} \mathrm{NExScI}$, California Institute of Technology, Pasadena, CA 91125, USA \\ ${ }^{7}$ National Radio Astronomy Observatory, Charlottesville, VA 22903-2475, USA \\ ${ }^{8}$ NASA Goddard Space Flight Center, Exoplanets and Stellar Astrophysics, Greenbelt, MD 20771, USA \\ Received 2010 December 15; accepted 2011 October 30; published 2012 February 1
}

\begin{abstract}
We present the first $N$-band nulling plus $K$ - and $L$-band $V^{2}$ observations of a young stellar object, MWC 325, taken with the $85 \mathrm{~m}$ baseline Keck Interferometer. The Keck nuller was designed for the study of faint dust signatures associated with debris disks, but it also has a unique capability for studying the temperature and density distribution of denser disks found around young stellar objects. Interferometric observations of MWC 325 at $K, L$, and $N$ encompass a factor of five in spectral range and thus, especially when spectrally dispersed within each band, enable characterization of the structure of the inner disk regions where planets form. Fitting our observations with geometric models such as a uniform disk or a Gaussian disk show that the apparent size increases monotonically with wavelength in the $2-12 \mu \mathrm{m}$ wavelength region, confirming the widely held assumption based on radiative transfer models, now with spatially resolved measurements over a broad wavelength range, that disks are extended with a temperature gradient. The effective size is a factor of about 1.4 and 2.2 larger in the $L$ band and $N$ band, respectively, compared to that in the $K$ band. The existing interferometric measurements and the spectral energy distribution can be reproduced by a flat disk or a weakly shadowed nearly flat disk model, with only slight flaring in the outer regions of the disk, consisting of representative "sub-micron" $(0.1 \mu \mathrm{m})$ and "micron" $(2 \mu \mathrm{m})$ grains of a 50:50 ratio of silicate and graphite. This is in marked contrast to the disks previously found in other Herbig Ae/Be stars, suggesting a wide variety in the disk properties among Herbig Ae/Be stars.
\end{abstract}

Key words: circumstellar matter - radiative transfer - stars: individual (MWC 325) - stars: pre-main sequence stars: variables: T Tauri, Herbig Ae/Be - techniques: interferometric

Online-only material: color figures

\section{INTRODUCTION}

The study of disks around pre-main-sequence (PMS) stars supports a long-term goal of the detection and characterization of exoplanets. The initial formation of a circumstellar disk is a consequence of the angular momentum distribution during star formation. For the first several million years, a PMS star is surrounded by a disk of gas and dust, which is a remnant reservoir left over from the build-up of stellar mass during the early stage of star formation. As the central star ages the disk disappears as a consequence of (1) accretion of disk material onto the central star, (2) ejection of material through stellar winds, (3) irradiation by stellar and other energetic photons, and (4) formation of planets. As circumstellar disks provide the raw material for planet formation, clues to the physical conditions of planet formation can be inferred from a detailed characterization, with the terrestrial planet zone corresponding to the inner regions of young stellar object (YSO) disks.

Herbig $\mathrm{Ae} / \mathrm{Be}(\mathrm{HAeBe})$ stars are a class of PMS stars of intermediate mass. The spectral types of HAeBe range from $B$ to $\mathrm{F}$, and their masses range from 2 to $8 M_{\odot}$ (Herbig 1960). The lifetime of the primordial disks of HAeBe stars is less than $\sim 3$ Myr (Hillenbrand et al. 1992; Hernández et al. 2005). These stars may be the progenitors of Vega-type debris stars.
A large number of HAeBe stars, across the luminosity range, have now been spatially resolved at near-infrared (Millan-Gabet et al. 1999, 2001; Eisner et al. 2003, 2004, 2007, 2009, 2010; Monnier et al. 2005, 2006; Malbet et al. 2007; Tatulli et al. 2007, 2011; Acke et al. 2008; Isella et al. 2008; Kraus et al. 2008a, 2008b, 2009; Tannirkulam et al. 2008a, 2008b; Ragland et al. 2009; Benisty et al. 2010a, 2010b, 2010c, 2011; Renard et al. 2010; Verhoeff et al. 2010; Weigelt et al. 2011) and mid-infrared (Tuthill et al. 2002; Leinert et al. 2004; Preibisch et al. 2006; Acke et al. 2008; Kraus et al. 2008b; Di Folco et al. 2009; Verhoeff et al. 2010) wavelengths using long baseline interferometry.

We have been carrying out a systematic investigation of YSO disks of different luminosity types through multi-color interferometry using the Keck Interferometer (KI). With $\sim 3$ mas angular resolution at $2.2 \mu \mathrm{m}$, the Keck Interferometer can resolve the inner circumstellar regions $(<1 \mathrm{AU}$ for distances $<300 \mathrm{pc}$ ) of PMS star disks where terrestrial planets can form. Our first results on MWC 419, based on simultaneous $K$ - and $L$-band measurements, were published in Ragland et al. 2009, where we found that MWC 419 exhibits relatively flat disk geometry. Prior to our work, multicolor interferometric observations at well-separated wavebands ( $K$ and $N$ bands) were reported for only two other HAeBe stars, MWC 297 (Acke et al. 2008) and MWC 147 (Kraus et al. 2008b), and more recently for 
HD 95881 (Verhoeff et al. 2010) using the Very Large Telescope Interferometer (VLTI). In this paper, we report observations of MWC 325 in the $K$ band $(2.0-2.4 \mu \mathrm{m}), L$ band $(3.5-4.1 \mu \mathrm{m})$, and $N$ band $(8-12 \mu \mathrm{m})$.

MWC 325 (V1295 Aql; HD 190073; HIP 98719) is classified as A2 IVev (Mora et al. 2001). An alternate spectral type of A2IIIpe can also be found in the literature (Schütz et al. 2009). The distance to MWC 325 is uncertain. The most commonly adopted distance in the literature has been $290 \mathrm{pc}$, based on the lower limit from Hipparcos measurements (van den Ancker et al. 1998). However, revised Hipparcos measurements (van Leeuwen 2007) suggest a lower limit of $340 \mathrm{pc}$. More recently, Montesinos et al. (2009) estimate a distance of $767_{+139}^{-76} \mathrm{pc}$ along with other stellar parameters such as mass, age, and luminosity through an iterative spectral energy distribution (SED) fitting process in conjunction with PMS tracks and isochrones. Their reported values for mass, luminosity, and age are $5.05_{+0.54}^{-0.47} M_{\odot}$, $470.8_{+189.2}^{-88.2} L_{\odot}$, and $0.6_{+0.2}^{-0.2} \mathrm{Myr}$, respectively. Those authors adopt an effective temperature and $\log g$ of $9500 \mathrm{~K}$ and $3.37 \pm$ 0.08 , respectively. Catala et al. (2007) adopt an effective temperature, $\log g$, and $\log \left(L / L_{\odot}\right)$ of $9250 \pm 250 \mathrm{~K}, 3.5 \pm$ 0.5 , and $1.9 \pm 0.12$, respectively, and obtain stellar mass, stellar radius, and age of $2.85 \pm 0.25 M_{\odot}, 3.6 \pm 0.5 R_{\odot}$, and $1.2 \pm 0.6 \mathrm{Myr}$, respectively, using theoretical evolutionary tracks. In this paper, we adopt an effective temperature, luminosity, radius, mass, and distance of $9250 \mathrm{~K}, 83 L_{\odot}, 3.6 R_{\odot}$, $2.85 M_{\odot}$, and $340 \mathrm{pc}$, respectively, for the central star. The reason for adopting a distance of $340 \mathrm{pc}$ is that our Kurucz model fits to $B V R I$ photometric data from the literature favors this value over the estimate of Montesinos et al. (2009) for a star on the main sequence. We discuss the impact of this choice on our results in Section 3.2.

MWC 325 shows a low-projected stellar rotational velocity, reported to be $9 \mathrm{~km} \mathrm{~s}^{-1}$ (Acke \& Waelkens 2004) to $12 \mathrm{~km} \mathrm{~s}^{-1}$ (Pogodin et al. 2005). The expected rotational velocity for an A2 IV/III star is in the range of 100-150 km s${ }^{-1}$ (Tassoul 2000). The observed low velocity suggests that the central star has either a low intrinsic rotational velocity or a typical intrinsic velocity but is viewed close to face-on.

MWC 325 exhibits a classical P Cygni II-type profile for the Balmer lines $\mathrm{H}_{\alpha}$ to $\mathrm{H}_{\delta}$ (Pogodin et al. 2005). These authors conclude that MWC 325 exhibits a strong stellar wind with an optically thick equatorial disk. Polycyclic aromatic hydrocarbon (PAH) emission was not detected in MWC 325 from a $3 \mu \mathrm{m}$ survey of HAeBe stars (Acke \& van den Ancker 2006).

In this paper, we report spectrally resolved $K-, L-$, and $\mathrm{N}$-band interferometric measurements of MWC 325. These probe physically different regions of the circumstellar disk having representative temperatures $\sim 1400 \mathrm{~K}, \sim 800 \mathrm{~K}$, and $\sim 300 \mathrm{~K}$, respectively, for simple blackbody grains. Moreover, these multi-wavelength observations probe different spatial scales as the fringe spacing on the sky is directly proportional to the wavelength. Thus, the $N$-band observations probe spatial scales roughly five times larger than that of the $K$-band observations. Discrete spatial distributions such as dust-rims and relatively smooth spatial distributions such as classical accretion disks are expected to have different size-versus-wavelength behaviors, and can be distinguished in such multi-color observations at well-separated wavelengths. MWC 325 has been spatially resolved previously by interferometers in the $H$ and $K$ bands (Millan-Gabet et al. 2001; Eisner et al. 2004, 2007, 2009; Monnier et al. 2006). These previous measurements enable a check for time variability in inner disk radii, which might be measurable given the dynamical time scale in this active region of the disk.

In Section 2, we present our observations and data reduction. In Section 3, we describe the data analysis where we fit the visibility-squared $\left(V^{2}\right)$ data with various YSO disk models. In Section 4 we discuss our results in conjunction with previous interferometric observations, and in Section 5 we provide a brief summary.

\section{OBSERVATIONS AND DATA REDUCTION}

$\mathrm{KI}$ is a near- and mid-infrared long-baseline interferometer consisting of two $10 \mathrm{~m}$ diameter telescopes separated by an $85 \mathrm{~m}$ baseline at a position angle (P.A.) of $\sim 38^{\circ}$ east of north, with the ability to null a central point source using phase control of the interfering beams. Both Keck telescopes are equipped with adaptive optics systems designed to compensate for atmospheric-induced wavefront aberrations, a crucial mitigation element for large-aperture long-baseline optical interferometry. The spatial resolution of our KI observations $(\lambda / 2 B)$ is $\sim 2.7$ mas, $\sim 4.5$ mas, and $\sim 12$ mas in the $K, L$, and $N$ bands, respectively. The spectral resolution is $R \sim 24,56$, and 21 over the $K, L$, and $N$ bands, respectively.

The observable in the $K$ and $L$ bands is visibility-squared, $V^{2}$ (the squared modulus of the visibility containing information about the spatial extent of the source), as a function of wavelength. The $V^{2}$ data in the $K$ and $L$ bands are collected simultaneously in the dual-band mode described by Ragland et al. $(2008,2009)$, and we refer to these as $K / L$ data below. In this mode, the Keck telescope pupils are split into halves, each with a separate beam train (this is an adaptation of the nuller configuration, described next) over the $85 \mathrm{~m}$ baseline. One beam train feeds a $K$-band $V^{2}$ system, which was the first KI science instrument, while the other feeds a more recently commissioned $L$-band $V^{2}$ system. Both systems operate similarly, with the observational scenario incorporating interleaved scans of calibrators, in addition to per-scan calibrations of the background and the flux ratio between the two apertures. The only significant difference between them is that for the $L$-band, because of the higher thermal background, nods to dark sky are required for the background measurement in order to achieve an accurate photometric calibration.

The $N$-band data are collected with the Keck Interferometer Nuller (KIN) described by Colavita et al. (2008, 2009). KIN operates differently than an ordinary $V^{2}$ instrument, with a different observable: the null leakage $l$. However, for relatively compact objects of small angular extent compared to the short baseline fringes (400 mas), the leakage is simply transformed to $V^{2}$ using the following equation (Koresko et al. 2006; Colavita et al. 2009) for comparison with the $K / L$ data:

$$
\begin{aligned}
V_{\text {measured }}^{2} & =\left[\frac{1-l}{1+l}\right]^{2} ; \\
\delta V_{\text {measured }}^{2} & =4\left[\frac{(1-l)}{(1+l)^{3}}\right] \delta l .
\end{aligned}
$$

Equation (1) assumes that the entire disk is much smaller than the KIN cross fringe, which has fringe spacing of 400 mas, and is a good approximation for these data.

The KIN uses the split pupil mode with two nulling beam combiners on the long $85 \mathrm{~m}$ baselines. In the data collection configuration, these two combiners are stabilized on a destructive fringe, ideally canceling all on-axis (point source) 
Table 1

Calibrated $V_{\text {total }}^{2}$ is Presented along with the Wavelength, $u v$ Points, and Measurement Errors

\begin{tabular}{|c|c|c|c|c|}
\hline $\begin{array}{l}\text { Wavelength } \\
(\mu \mathrm{m})\end{array}$ & $\begin{array}{c}u \\
(\mathrm{~m})\end{array}$ & $\begin{array}{c}v \\
(\mathrm{~m})\end{array}$ & $V_{\text {total }}^{2}$ & $V_{\text {total }}^{2}$ Error \\
\hline \multicolumn{5}{|c|}{ Spectrally dispersed KIN measurements (UT 2008 August 19) } \\
\hline 2.004 & 54.548 & 64.593 & 0.1610 & 0.0419 \\
\hline 2.102 & 54.548 & 64.593 & 0.1519 & 0.0414 \\
\hline 2.169 & 54.548 & 64.593 & 0.1469 & 0.0411 \\
\hline 2.288 & 54.548 & 64.593 & 0.1445 & 0.0409 \\
\hline 2.377 & 54.548 & 64.593 & 0.1448 & 0.0407 \\
\hline 8.209 & 54.548 & 64.593 & 0.4389 & 0.0073 \\
\hline 8.765 & 54.548 & 64.593 & 0.4784 & 0.0104 \\
\hline 9.186 & 54.548 & 64.593 & 0.5038 & 0.0068 \\
\hline 9.730 & 54.548 & 64.593 & 0.5234 & 0.0083 \\
\hline 10.270 & 54.548 & 64.593 & 0.5359 & 0.0108 \\
\hline 10.726 & 54.548 & 64.593 & 0.5579 & 0.0098 \\
\hline 11.232 & 54.548 & 64.593 & 0.5728 & 0.0142 \\
\hline 11.741 & 54.548 & 64.593 & 0.5747 & 0.0219 \\
\hline 12.223 & 54.548 & 64.593 & 0.6096 & 0.0390 \\
\hline 12.713 & 54.548 & 64.593 & 0.6294 & 0.0859 \\
\hline \multicolumn{5}{|c|}{ Spectrally dispersed KI measurements (UT 2009 October 27) } \\
\hline 2.004 & 22.261 & 68.356 & 0.1720 & 0.0408 \\
\hline 2.102 & 22.261 & 68.356 & 0.1686 & 0.0400 \\
\hline 2.169 & 22.261 & 68.356 & 0.1664 & 0.0400 \\
\hline 2.288 & 22.261 & 68.356 & 0.1622 & 0.0400 \\
\hline 2.377 & 22.261 & 68.356 & 0.1597 & 0.0400 \\
\hline 3.487 & 22.014 & 68.367 & 0.2512 & 0.0400 \\
\hline 3.568 & 22.014 & 68.367 & 0.2645 & 0.0400 \\
\hline 3.629 & 22.014 & 68.367 & 0.2761 & 0.0401 \\
\hline 3.734 & 22.014 & 68.367 & 0.2833 & 0.0401 \\
\hline 3.808 & 22.014 & 68.367 & 0.2931 & 0.0401 \\
\hline 3.846 & 22.014 & 68.367 & 0.3041 & 0.0401 \\
\hline 3.978 & 22.014 & 68.367 & 0.3163 & 0.0401 \\
\hline 4.029 & 22.014 & 68.367 & 0.3291 & 0.0402 \\
\hline \multicolumn{5}{|c|}{ Broadband KI measurements (UT 2008 August 19) } \\
\hline 2.180 & 55.239 & 64.337 & 0.1435 & 0.0412 \\
\hline \multicolumn{5}{|c|}{ Broadband KI measurements (UT 2009 October 27) } \\
\hline 2.180 & 22.261 & 68.356 & 0.1735 & 0.0412 \\
\hline \multicolumn{5}{|c|}{ Broadband CHARA measurements (UT 2010 July 16) } \\
\hline 2.130 & 58.888 & 28.609 & 0.2303 & 0.0398 \\
\hline
\end{tabular}

light, and only transmitting the extended emission. The leakage from these two nulling beam combiners is combined in a third beam combiner, which uses fast fringe scanning to measure the leakage flux in the presence of the large $N$-band background. By changing the track point on the nulling beam combiners from destructive interference to constructive interference, a "photometric" measurement can be made to normalize the leakage flux, producing the main observable, the (normalized) null leakage $l$. Because the achievable control bandwidth using just the $N$-band light is lower than that needed to compensate for atmospheric turbulence, KIN also uses two $K$-band systems on the long baselines for fringe stabilization. However, as these systems provide $K$-band $V^{2}$ (with a resolution of four channels across the $K$ band) as an auxiliary data product, both $K$ - and $N$-band data can be collected simultaneously, and we refer to these as $K / N$ data, below. The observational scenario for KIN is similar to other modes, using interleaved calibrators to measure the system leakage, in the same way interleaved calibrators measure the system visibility for $V^{2}$ observations.

The field-of-view of the $K$ - and $L$-band instruments is defined by the single mode fibers that couple light to the HAWAII
$(K$-band) and PICNIC ( $L$-band) infrared detectors, and that of KIN is defined by the pinhole used in the $N$-band camera. The resultant field-of-view (FWHM) is $\sim 60$ mas for $K$-band, $\sim 100$ mas for the $L$-band, and $\sim 500$ mas for $N$-band measurements. These field restrictions were used in our modeling work.

The observations reported here were taken on the nights of UT 2008 August 19 ( $K$ - and $N$-band measurements) and UT 2009 October 27 ( $K$ - and $L$-band measurements). We observed five calibrators-HD 188310, HD 193579, HD 206445, HD 190007, and HD187691 - under similar observing conditions as the science target. We performed bracketed calibration-meaning that our observing sequence consisted of calibrator-target-calibrator measurements. The adopted angular diameters of the calibrators are $2.0 \pm 0.2,2.0 \pm 0.2,1.8 \pm 0.2,0.5 \pm 0.1$, and $0.6 \pm 0.1 \mathrm{mas}$, respectively (van Belle 1999). We cross-checked these diameters through SED model fits to photometric data available in the literature. Table 1 lists the calibrated visibility-squared measurements $\left(V_{\text {total }}^{2}\right)$.

The measurements presented in this paper were taken over a narrow range of P.A.s $\left(39^{\circ}-41^{\circ}\right.$ and $17^{\circ}-19^{\circ}$ east of north) 
Table 2

Calibrated $K$-band (Broadband) $V_{\text {total }}^{2}$ Presented along with $u v$ Points and Measurement Errors for KI and PTI Archive Data

\begin{tabular}{|c|c|c|c|c|c|c|}
\hline UT Date & $\begin{array}{c}u \\
(\mathrm{~m})\end{array}$ & $\begin{array}{c}v \\
(\mathrm{~m})\end{array}$ & $V_{\text {total }}^{2}$ & $V_{\text {total }}^{2}$ Error & Calibrators & $\begin{array}{l}\text { Calibrator Diameter } \\
\text { (mas) }\end{array}$ \\
\hline \multicolumn{7}{|c|}{ PTI Archive } \\
\hline \multirow[t]{3}{*}{2003 Oct 13} & -77.805 & -24.805 & 0.1833 & 0.0313 & HD 174160 & $0.38 \pm 0.01$ \\
\hline & & & & & HD 187923 & $0.80 \pm 0.21$ \\
\hline & & & & & HD 193556 & $0.73 \pm 0.09$ \\
\hline \multirow[t]{2}{*}{2003 Oct 14} & -48.412 & 65.934 & 0.1815 & 0.0333 & HD 187923 & $0.80 \pm 0.21$ \\
\hline & & & & & HD 193556 & $0.73 \pm 0.09$ \\
\hline \multicolumn{7}{|c|}{ KI Archive } \\
\hline \multirow[t]{8}{*}{2007 Jul 2} & 40.935 & 67.021 & 0.1539 & 0.0313 & HD 183324 & $0.26 \pm 0.01$ \\
\hline & & & & & & $0.64 \pm 0.01$ \\
\hline & 32.564 & 67.769 & 0.1861 & 0.0318 & HD 183385 & $0.26 \pm 0.01$ \\
\hline & & & & & & $0.64 \pm 0.01$ \\
\hline & 25.758 & 68.181 & 0.2046 & 0.0350 & HD 183385 & $0.26 \pm 0.01$ \\
\hline & & & & & & $0.64 \pm 0.01$ \\
\hline & 20.092 & 68.438 & 0.2179 & 0.0344 & HD 183385 & $0.26 \pm 0.01$ \\
\hline & & & & & & $0.64 \pm 0.01$ \\
\hline \multirow[t]{4}{*}{2009 Jul 16} & 55.361 & 63.159 & 0.1040 & 0.0312 & HD 183926 & $0.35 \pm 0.04$ \\
\hline & & & & & HD 187182 & $0.33 \pm 0.04$ \\
\hline & & & & & HD 194244 & $0.30 \pm 0.10$ \\
\hline & & & & & HD 190067 & $0.36 \pm 0.01$ \\
\hline
\end{tabular}

and projected baselines $(83.9-84.9 \mathrm{~m}$ and 71.6-72.1 $\mathrm{m}$ ) for the $K / N$ and $K / L$ measurements, respectively.

We also carried out follow-up broadband observations of MWC 325 with the CHARA array in the $K$ band using the Classic beam-combiner and the E2-E1 $(65.917 \mathrm{~m})$ baseline at P.A. $65^{\circ}$ on 2010 July 16 . The calibrators used for these observations are HD188107, HD192343, and HD188953, and the adopted angular diameters are $0.186 \pm 0.005,0.206 \pm 0.005$, and 0.287 \pm 0.004 mas, respectively. We used these observations, in conjunction with our KI broadband ( $K$-band) observations (Table 1 ) and $K$-band measurements in the literature (Table 2), to determine the inner-rim diameter and the inclination angle of the disk in the later part of Section 3.1.

As the spectrally dispersed $\mathrm{K} / \mathrm{N}$ and $\mathrm{K} / \mathrm{L}$ observations were taken at different epochs - separated by $\sim 14$ months - variability is an obvious concern when we combine these two sets of data. Since $K$-band measurements were taken on both occasions, we use these data to investigate possible variability. The mean $K$-band squared visibility was $0.14 \pm 0.04$ and $0.17 \pm 0.04$ for the epochs UT 2008 August 19 and UT 2009 October 27, respectively. These $K$-band $V^{2}$ measurements for the two epochs are comparable to within the $1 \sigma$ measurement errors. However, the baselines for these two epochs are somewhat different, complicating direct comparison of these two measurements. Comparing these measurements with the $K$-band measurements in the literature (Table 2), taken during 2003-2010, shows no evidence of size variability. Eisner et al. (2007), who were specifically looking for interferometric variability evidence in a small sample of YSOs, did not find any detectable size variations over time. We conclude, given the precision of our present observations, that there is no detectable size variability between these two epochs.

\section{ANALYSIS}

The measured $V_{\text {total }}^{2}$ includes contributions from the central star $\left(V_{*}^{2}\right)$ and the circumstellar disk $\left(V_{\text {disk }}^{2}\right)$. For the modeling analysis presented in this section, the visibility-squared of the circumstellar disk of MWC 325 is obtained from the measured data by removing the contributions from the central star as follows. As the light from the central star and the circumstellar disk is incoherent, the total complex visibility is the sum of the complex visibilities of the star and the disk, i.e.,

$$
\widehat{V}_{\mathrm{tot}}=\frac{F_{*} \widehat{V}_{*}+F_{\mathrm{disk}} \widehat{V}_{\mathrm{disk}}}{F_{*}+F_{\mathrm{disk}}},
$$

where $F_{*}$ and $F_{\text {disk }}$ are the stellar and disk fluxes, respectively. The squared modulus of this equation can be written in quadratic form as

$$
V_{\text {disk }}^{2}+2 r V_{*} V_{\text {disk }} \cos \theta+r^{2} V_{*}^{2}-(1+r)^{2} V_{\text {tot }}^{2}=0,
$$

where $r=F_{*} / F_{\text {disk, }}, V_{*}=\left|\tilde{V}_{*}\right|, V_{\text {disk }}=\left|\tilde{V}_{\text {disk }}\right|$, and $\theta$ is the phase difference between the star and disk complex visibilities. The phase shift $\theta$ includes both the effective photocenter shift measured at the spatial frequency sampled by the interferometer, as well as the difference in the sign of the visibilities between the two components (for example, if one object were sampled on a negative lobe of its visibility function). If the disk and star are symmetric, and they are sampled on the main lobe of their visibility functions (i.e., they are reasonably compact), then $\theta=$ $2 \pi B \cdot \delta / \lambda$, where $\delta$ is the angular photocenter separation of the disk and star; this is the case in this paper. The solutions of this quadratic equation are

$$
V_{\mathrm{disk}}=-r V_{*} \cos \theta \pm\left[(1+r)^{2} V_{\mathrm{tot}}^{2}-r^{2} V_{*}^{2} \sin ^{2} \theta\right]^{\frac{1}{2}} .
$$

If the star and disk share the same effective photocenter, and they are both sampled on the main lobe of their visibility functions, then this can be written in terms of fringe amplitudes as

$$
V_{\mathrm{disk}}^{2}=\left[(1+r) V_{\mathrm{tot}}-r V_{*}\right]^{2} \text {. }
$$

The stellar flux contribution in the $K, L$, and $N$ bands is estimated by fitting the Kurucz stellar atmospheric model (Kurucz 1970) 
Wavelength Dependent Disk Size of MWC 325
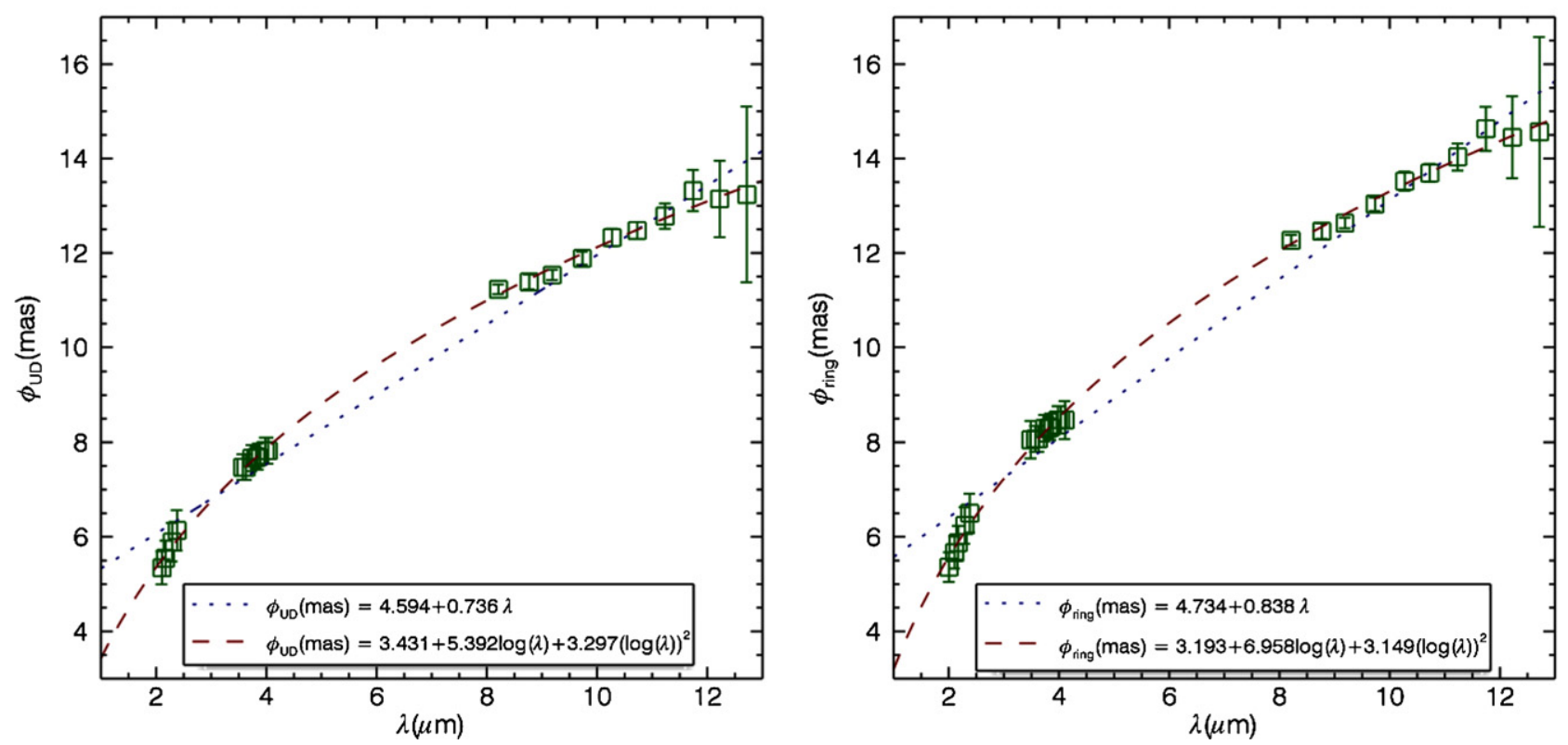

Figure 1. Wavelength-dependent fits to the observed data. The estimated stellar contribution has been subtracted from the measurements. Left: observed uniform-disk angular diameters as a function of wavelength are shown along with error bars. Linear (dotted, $\left.\chi_{R}^{2}=3.0\right)$ and log-quadratic (dashed, $\left.\chi_{R}^{2}=0.3\right)$ fits to these diameters are also shown. Right: same, except for ring model. The $\chi_{R}^{2}$ values for linear and log-quadratic model fits are 3.7 and 0.3 , respectively.

(A color version of this figure is available in the online journal.)

for an A2-type star of solar metallicity $\left(T_{\text {eff }}=9250 \mathrm{~K}, \log g=\right.$ 3.5 ) to dereddened $B V R$ photometric fluxes (see below for the adopted interstellar extinction) and extrapolating the stellar atmospheric model at desired infrared wavelengths. The derived star-to-disk flux ratio, $r$, is $0.2419,0.0677$, and 0.0086 in the $K, L$, and $N$ bands, respectively. We use Equation (5) for the modeling work presented in Sections 3.1 and 3.2, and for the initial modeling of Section 3.3. When the star and the disk do not share the same photocenter, and assuming symmetric, reasonably compact objects, Equation (5) will overcorrect for the effect of the star, resulting in a lower estimated $V_{\text {disk }}^{2}$. For the final radiative transfer models presented in Section 3.3, we account for the photocenter effect (see Section 3.3 for the details).

The central star is assumed to be unresolved for our observations (i.e., $V_{*}^{2}=1.0$; the expected angular diameter of the central star is 0.12 mas for the assumed stellar radius and the distance, resulting in visibility-squared of $>0.999$ for our instrument configurations). These central star corrections yield $V_{\text {disk }}^{2}$ values that are smaller than the total $V^{2}$ by $0.09(\sim 60 \%), 0.03(\sim 12 \%)$, and $0.003(\sim 0.6 \%)$ in $K, L$ and $N$ bands, respectively. Such corrections are most important at the shortest wavelengths where the star is the brightest and the disk-to-star ratio is the smallest.

For our SED analysis, photometric measurements from the literature (2MASS All-Sky Catalog of Point Sources (Skrutskie et al. 2006); The Hipparcos and Tycho Catalogs (ESA 1997); IRAS catalog of Point Sources, Version 2.0 (IPAC 1986); Akari/IRC mid-IR all-sky Survey and Akari/FIS All-Sky Survey Point Source Catalogs (ISAS/JAXA 2010; Tannirkulam et al. 2008a) are corrected for interstellar extinction using the extinction law of Cardelli et al. (1989). The extinction for MWC 325 in the $V$ band is assumed to be $A_{v}=0.19$ (Van den Ancker et al. 1998). We also used Infrared Space Observatory Short-Wavelength Spectrometer in the 2.36-4.1 $\mu \mathrm{m}$ wavelength region (Vandenbussche et al. 2002) and Spitzer spectra from the Spitzer Space Telescope in our SED modeling.

Our $K$-band $V^{2}$ measurements (Table 1 ) are consistent with earlier broadband $K$ measurements and provide additional spectrally resolved information at different P.A.s, enabling the determination of disk inclination angle. Our $L$-band and $N$-band measurements are unique and are also spectrally resolved.

\subsection{Simple Wavelength-Dependent Geometrical Models}

In this section, we use our spectrally dispersed data within the $K-, L$-, and $N$-band wavelength regions to investigate wavelength dependency of the source size and broadband ( $K$-band) measurements to constrain the inclination angle of the disk. We chose three geometrical models, namely, uniformdisk, Gaussian distribution, and ring models to fit our spectrally dispersed measurements. The equations for these geometric models are given by Ragland et al. (2009), who showed that the wavelength variation of the object size over the $K$ and $L$ bands was needed to fit the visibilities for MWC 419. However, we begin here for the current case, MWC 325, with the simplest possible wavelength-independent geometrical model and then motivate the need for a more complex model.

For wavelength-independent models, the derived uniformdisk, Gaussian, and ring angular sizes from simultaneous fits to our multi-wavelength measurements are 11.4 mas, 6.9 mas, and 12.7 mas, respectively. The corresponding reduced $\chi^{2}$ $\left(\chi_{R}^{2}\right)$ values are 39,28 , and 45 , respectively. As indicated by the very poor $\chi_{R}^{2}$ values, the wavelength-independent uniform disk, Gaussian, and ring models all fail to fit multi-wavelength visibilities in the $K, L$, and $N$ bands.

Next we fit the data with wavelength-dependent sizes, taking advantage of the spectrally dispersed data across each of our bands. The resulting size-wavelength dependence for the uniform disk model in the $2-12 \mu \mathrm{m}$ regions can be fit with a simple linear relationship, $\phi_{\mathrm{UD}}(\mathrm{mas})=4.594+0.736 \lambda$, with a $\chi_{R}^{2}$ of 3.0. Increasing the complexity of the model even further, a log-quadratic fit of the form, $\phi_{\mathrm{UD}}$ (mas) $=$ $3.431+5.392 \times \log (\lambda)+3.297 \times[\log (\lambda)]^{2}$, to the derived apparent wavelength-dependent diameters (Figure 1, left panel) gives a $\chi_{R}^{2}$ of 0.3 . The purpose of using an arbitrary function 
Table 3

Derived Parameters for the Disk Models Presented in Section 3.2

\begin{tabular}{lccc}
\hline \hline Model Parameters & Face-on Accretion Disk & Face-on Power-law Disk & Inclined Power-law Disk \\
\hline$R_{\text {in }}(\mathrm{AU})$ & $0.68 \pm 0.01$ & $0.66 \pm 0.02$ & $0.76 \pm 0.05$ \\
$T_{\text {in }}(\mathrm{K})$ & 628 & 1500 (fixed) & 1500 (fixed) \\
Radial power-law exponent, $\alpha$ & 0.75 (fixed) & $1.12 \pm 0.02$ & $1.26 \pm 0.05$ \\
Disk inclination, $i$ & $0^{\circ}$ (fixed) & $0^{\circ}$ (fixed) & $72^{\circ} \pm 4^{\circ}$ \\
Position angle of the major-axis & $\ldots$ & $\ldots$ & $49^{\circ} \pm 4^{\circ}$ \\
$M_{\text {acc }}\left(M_{\odot} \mathrm{yr}^{-1}\right)$ & $3 \times 10^{-6}$ & $\ldots$ & $\ldots$ \\
$\chi_{\text {IF }}^{2}$ & 2.6 & 1.3 & 0.6 \\
$\chi_{\text {SED }}^{2}$ & 9.8 & 126.0 & 5.9 \\
$\chi_{\text {Total }}^{2}$ & 8.0 & 94.9 & 4.6 \\
\hline
\end{tabular}

Note. The entries $\chi_{\mathrm{IF}}^{2}, \chi_{\mathrm{SED}}^{2}$, and $\chi_{\text {Total }}^{2}$ refer to the $\chi_{R}^{2}$ for interferometric data, SED data, and the combined set of interferometric and SED data, respectively.

here is to get a rough knowledge of the size dependence as a function of wavelength. For comparison, size-wavelength dependence for a ring disk model is also shown in Figure 1 (right panel).

The models presented above assume face-on geometry for the disk consistent with the low $v \sin (i)$ measured for the star if the star and disk angular momentum axes are aligned. A disk with an inclination angle $i$ could have different properties, notably a larger inner disk radius, depending on the inclination angle and the P.A. of the disk relative to the direction of the projected baseline, hence fringes.

While multi-wavelength observations in the near- and midinfrared wavelengths are effective in probing the extended disk, single wavelength measurements as a function of P.A. could be used to determine the inclination angle of the disk. We used interferometric measurements in the $K$ band for this purpose to define the geometry of the inner-rim of the disk. We adopted an elliptical ring to represent the inner-rim and carried out model fit to our broadband $K$-band measurements from KI and CHARA (Table 1). We also included archived $K$-band interferometric measurements from KI (Keck Interferometer Archive, NExScI) on 2007 July 2 (P.A.: 31.42, 25.67, 21.92, 19.89; baseline: 78.53, 75.19, 73.38, $72.50 \mathrm{~m}$ ) and 2009 July 16 (P.A.: 41.24; baseline: $83.99 \mathrm{~m}$ ), and from PTI (Palomar Testbed Interferometer Archive, NExScI) on 2003 October 13 (P.A.: 72.32; baseline: $81.66 \mathrm{~m}$ ) and 2003 October 14 (P.A.: 143.71; baseline: $81.80 \mathrm{~m}$ ) in Table 2. The best-fit model is shown in Figure 2. The derived ring diameter, inclination angle, and P.A. of the major axis of the disk (east of north) are $5.85 \pm$ $0.2 \times 5.56 \pm 0.2 \mathrm{mas}, 18_{+30}^{-18} \mathrm{deg}$, and $142_{+30}^{-30} \mathrm{deg}$, respectively. In the following section, we explore more detailed disk models to explain our observations.

\subsection{Geometrically Thin, Optically Thick Disk Models}

Moving from simple geometric to more physically realistic models, we consider first a face-on accretion disk model (Hillenbrand et al. 1992) with a radial temperature distribution of the form $T(r) \propto r^{-\left(\frac{3}{4}\right)}$, where $r$ is the radial distance from the central star. The visibilities are computed by numerically summing the contributions from annular rings of infinitesimally small widths and weighting them by their respective flux contributions. We fit visibility and SED data simultaneously by treating the inner disk (hole) radius and the accretion rate as freely varying model parameters.

The best-fit model parameters are given in Table 3 and the fits are shown in Figure 3. Even though the model itself is ax-

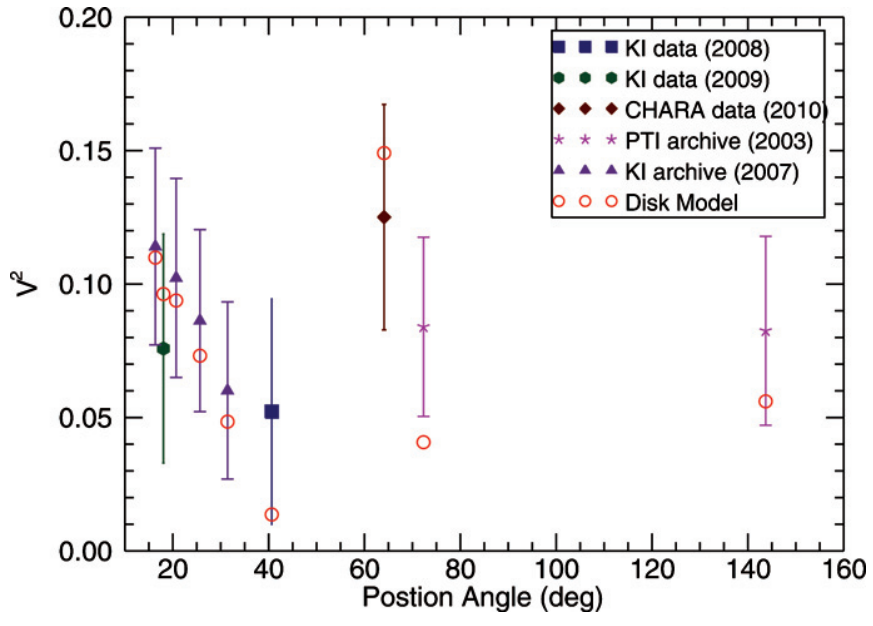

Figure 2. Elliptical ring model fit to $K$-band interferometric data. (A color version of this figure is available in the online journal.)

isymmetric, since the effective projected baselines and P.A.s of the two epoch observations are different, two corresponding model curves are shown in Figure 3 (left panel). Notably, the classical accretion disk model fails to fit simultaneously the interferometric and the SED data. As illustrated in Figure 3 , the fit to the interferometric data vastly underpredicts the amount of flux needed between 2 and $11 \mu \mathrm{m}$ in the SED $\left(\chi_{\mathrm{IF}}^{2}=2.6, \chi_{\mathrm{SED}}^{2}=9.8\right.$, and $\left.\chi_{\mathrm{Total}}^{2}=8.0\right) \cdot \chi_{\mathrm{Total}}^{2}$ is computed combining both squared-visibilities and SED data with their measurements errors. Specifically, the derived inner disk temperature is $628 \mathrm{~K}$ while the SED requires hotter dust. The derived inner angular diameter of the face-on disk is $4.02 \pm$ 0.04 mas. The fit to the interferometric data points could be improved $\left(\chi_{\mathrm{IF}}^{2}=1.1\right)$ by changing the inclination angle of the accretion disk to $70^{\circ}$ and the mass accretion rate to $8 \times$ $10^{-7} M_{\odot} \mathrm{yr}^{-1}$. However, such a model highly underestimates SED flux $\chi_{\mathrm{SED}}^{2}=28.3$ and $\chi_{\text {Total }}^{2}=21.5$, particularly in the near- to mid-infrared from about 1 to $10 \mu \mathrm{m}$.

One way to get hotter dust with only small changes to the size of the inner hole is to steepen the disk temperature gradient. We thus fit our data with a power-law temperature gradient model of functional form $T(r) \propto r^{-\alpha}$, where $r$ is the radial distance from the central star and $\alpha$ is the power-law parameter $(0.75$ for the classical accretion disk). The temperature of the inner edge of the dust disk is fixed at $1500 \mathrm{~K}$, corresponding to a notional dust destruction radius. The inner radius and $\alpha$ are treated as free model parameters. The inclination angle of the disk is fixed 

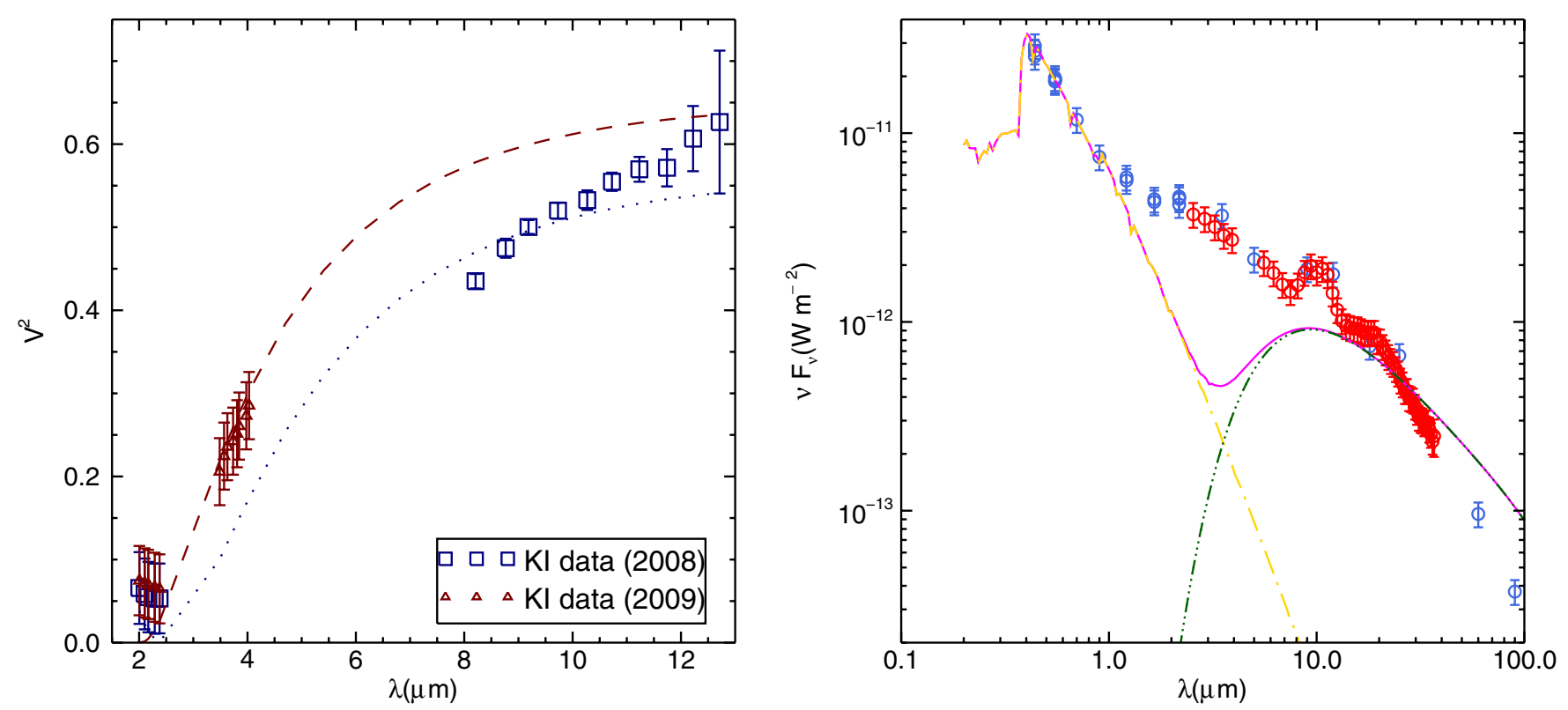

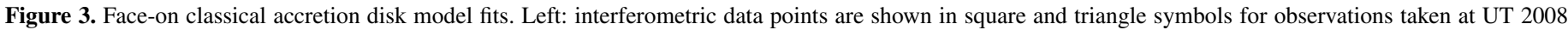

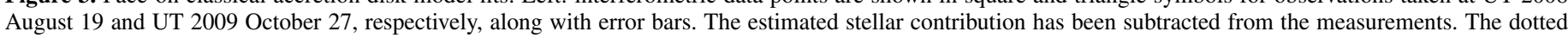

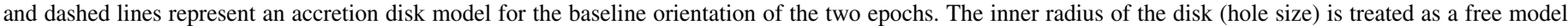

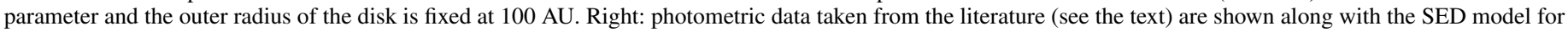
the same accretion disk model. The dash-dotted, triple-dot-dashed, and solid lines are model SEDs of the star, disk, and star-plus-disk, respectively.

(A color version of this figure is available in the online journal.)
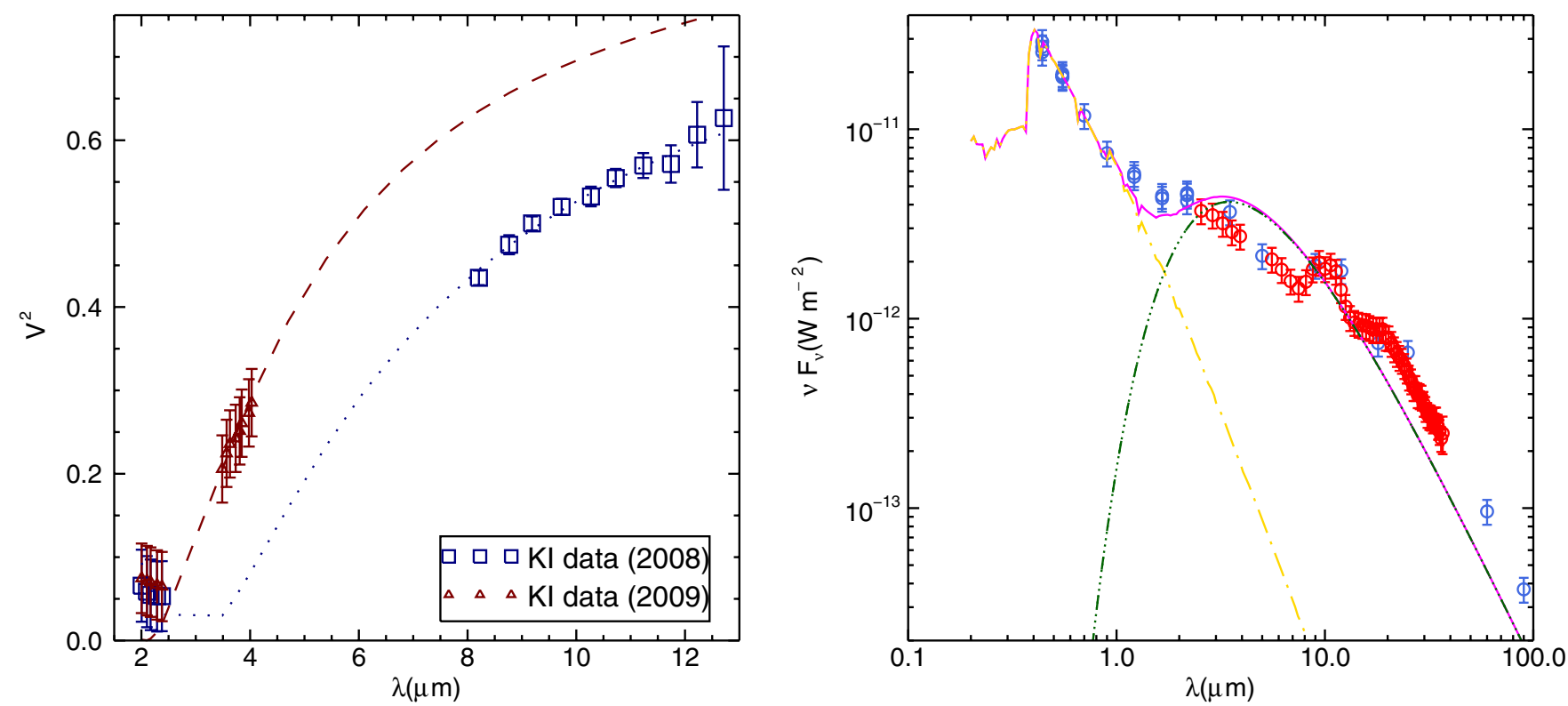

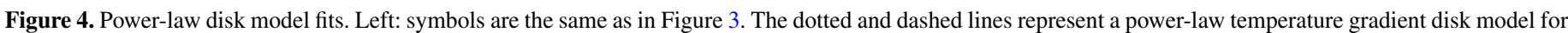

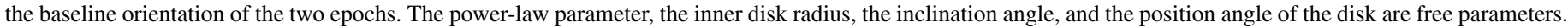

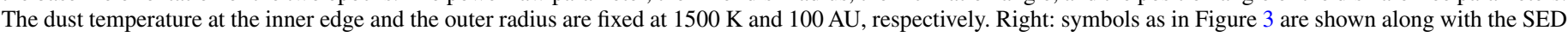
model for the same power-law model. The dash-dotted, triple-dot-dashed, and solid lines are model SEDs of the star, disk, and star-plus-disk, respectively.

(A color version of this figure is available in the online journal.)

to zero in the case of a face-on power-law disk, but varied as a free parameter in the case of an inclined power-law disk.

The resultant model fit (in the case of inclined power-law disk) is shown in Figure 4 and the model parameters are given in Table 3. The derived angular diameter of the inner dust edge along the major axis and the power-law exponent are $3.86 \pm$ 0.10 mas and $1.12 \pm 0.02$ for the face-on disk, and $4.47 \pm$ 0.20 mas and $1.26 \pm 0.05$ for the inclined disk, respectively. The inclination angle of the disk is $\sim 72^{\circ} \pm 4^{\circ}$ and the P.A. of the major axis of the disk is $\sim 49^{\circ} \pm 4^{\circ}$. While our model fit to the interferometric and SED data are improved over the classical accretion disk model, the SED fit underpredicts the 1-3 $\mu \mathrm{m}$ and 10-60 $\mu \mathrm{m}$ flux, and overpredicts the 3-9 $\mu \mathrm{m}$ flux. This parameterized disk model is meant to approximate the emission near the "inner dust rim" disk component, which dominates the infrared emission, and which for this type of star has been found to often be required in order to explain the spatially resolved IR observations (see, e.g., the recent review by 
Dullemond \& Monnier 2010). The seemingly steep temperature gradients derived from these power-law models are discussed in Section 4.

As we mentioned before, the distance to MWC 325 is uncertain. We adopted a distance of $340 \mathrm{pc}$ as the blackbody model fits to $B V R I$ photometric data favors this value. If we were to use 767 pc (Montesinos et al. 2009), the inner radius of the disk would have a larger value for the accretion disk and the power-law models. The resultant inner radius of the face-on accretion disk, face-on power-law, and inclined power-law models are $1.51 \pm 0.01 \mathrm{AU}, 1.48 \pm 0.02 \mathrm{AU}$, and $1.71 \pm 0.05 \mathrm{AU}$, respectively. While the other parameters of the power-law models are not affected due to this distance change, some parameters of the face-on accretion disk model changed; the temperature of the inner edge decreased to $645 \mathrm{~K}$ and the mass accretion rate increased to $4 \times 10^{-5} M_{\odot} \mathrm{yr}^{-1}$. The parameters of the disk models presented in this section should be treated with some caution, especially the inclination angle (and hence P.A.) and mass accretion rate, which are arbitrarily used to scale the disk flux in order to match the overall flux level of the SED data. However, they do not fit the SED data satisfactorily. In the following section, we present a more complex physical model based on radiative transfer calculations to improve fit to our interferometric measurements and SED data.

\subsection{Radiative Transfer Models}

We have also carried out two-dimensional radiative transfer modeling of the dusty disk of MWC 325 through Monte Carlo simulations (Ohnaka et al. 2006) in order to explain our interferometric measurements. In this radiative transfer calculation, photon packets randomly released from the stellar surface are subjected to absorption and scattering by multiple species of dust grains in the circumstellar disk (we assumed isotropic scattering for simplicity). The dust temperature of each grain species is computed using the method of Bjorkman \& Wood (2001). The outputs of the Monte Carlo code are the SED viewed from arbitrary inclination angles, the temperature of each grain species, and the monochromatic mean intensity at each cell position in the dust disk. Using resultant mean intensity and dust temperatures, monochromatic images viewed from any arbitrary angle are computed through ray tracing.

We used a Kurucz stellar atmospheric model (Kurucz 1970) for an A2-type star of solar metallicity $\left(T_{\text {eff }}=9250 \mathrm{~K}, \log g=\right.$ 3.5) as our input stellar spectrum for our radiative transfer calculations. We assumed that the disk consists of a mixture of graphite and silicate with equal fractional abundances and used the optical properties presented by Draine \& Lee (1984). For the modeling of MWC 325, we adopted representative "sub-micron" $(0.1 \mu \mathrm{m})$ and "micron" $(2 \mu \mathrm{m})$ radius grains. We computed models with different grain size distributions, for example, models only with the sub-micron-sized grains or micron-sized grains, as well as models with millimeter-sized grains. However, it turned out that a two-grain model consisting of 0.1 and $2 \mu \mathrm{m}$ grain is needed to reproduce the observed SED and interferometric data.

The dust density distribution in the circumstellar disk is characterized by the standard flared disk geometry given by

$$
\begin{gathered}
\rho_{i}(r, z) \propto\left(\frac{r}{r_{0}}\right)^{-p} \exp \left[-\frac{1}{2}\left(\frac{z}{h_{i}(r)}\right)^{2}\right] \\
h_{i}(r)=h_{i, 0}\left(\frac{r}{r_{0}}\right)^{q},
\end{gathered}
$$

where $r$ is the radial distance in the equatorial plane, $z$ is the height from the equatorial plane, and $h_{i, 0}$ is the scale height of the $i$ th grain species at some reference radius $r_{0}$. In the region with $r$ smaller than the inner boundary radius, dust was assumed to be absent.

In order to allow for the vertical dust segregation that has been inferred (Tannirkulam et al. 2007), we treated the scale heights of the small and large grains as free parameters. The other free parameters are the disk's inner boundary radius of the dust grains (assumed to be the same for both grain species) and the optical depth of dust grains (equivalent to specifying the mass of dust grains).

The computation is CPU-intensive and performed in a parallel computing environment, simultaneously running on three Sun Fire V440 servers (each one powered by four $1.593 \mathrm{GHz}$ UltraSPARC IIIi processors), taking about two days of CPU time to generate a single model with adequate signal-to-noise ratio $(\mathrm{S} / \mathrm{N})$, spatial resolution, and field of view. We used 2 $\times 10^{7}$ photon packets for most of our simulations for stable results with adequate $S / N$. A library of model images at the wavelengths of interest and the corresponding model SEDs are generated for a range of model parameters. Model $V^{2}$ values are computed from these images and compared with measured $V^{2}$ data by varying the P.A. of the disk through nonlinear leastsquares fitting. The choice of distance has no impact on our radiative transfer modeling since the raw images are in units of stellar radius and are scaled to fit the SED data. The scaling of these images gives a distance of $321 \mathrm{pc}$ to MWC 325 within roughly $5 \%$ of the adopted 340 pc value.

Our attempt to fit simultaneously interferometric and SED data with a flared-disk model $(q \geqslant 1)$ was not successful. In these models, the outer region of the disk is efficiently warmed up so that the $10 \mu \mathrm{m}$ silicate emission originates in the outer region of the disk. This makes the object appear larger in the $10 \mu \mathrm{m}$ silicate feature than at 8 or $13 \mu \mathrm{m}$. However, as shown in Figure 1, the size of the object increases monotonically from 8 to $13 \mu \mathrm{m}$ without regard to the presence of the $10 \mu \mathrm{m}$ feature, whether in emission or absorption. The situation is different from that of typical studies of gas in circumstellar disks in which the visibilities across $\mathrm{H}$ and $\mathrm{CO}$ features change relative to the continuum, allowing conclusions on the relative spatial distribution of gas and dust. We conclude that flared disk models with $q \geqslant 1$ cannot explain the observed $N$-band visibilities.

Subsequently, we explored the parametric space, $q<1$ and the best fit provides $q=0.875$, suggesting that the disk is fairly flat with little or no flaring. A comparison between our observations and the best-fit model is shown in Figure 5. The best fit to the data can be obtained by a disk model viewed from an intermediate inclination angle of $45^{\circ}$ with a dust sublimation radius of $1.26 \mathrm{AU}$.

As mentioned before, if the photocenters of the star and disk are not the same, the use of Equation (5) incorrectly estimates the squared visibility of the disk. We use Equation (5) for the initial models. Then we derive fringe phase information from the model images to compute an improved set of squared visibilities for the disk using Equation (4). We iterate this procedure a few times until it converges. The maximum correction required for the photocenter effect was 0.035 in squared visibility-well within the $1 \sigma$ measurement errors. The assumed stellar parameters are given in Table 4 and the characteristics of the flat-disk are given in Table 5. Figure 5 (left) shows that the interferometric data spanning from 2 to $13 \mu \mathrm{m}$ are reproduced well by this model. Figure 5 (right) shows that the near-IR part of the 
Table 4

Assumed Stellar Parameters

\begin{tabular}{lc}
\hline \hline Stellar Parameters & Value \\
\hline Spectral type & A2 IVev \\
Stellar luminosity & $83 L_{\odot}$ \\
Stellar effective temperature & $9250 \mathrm{~K}$ \\
Stellar radius & $3.6 R_{\odot}$ \\
Stellar mass & $2.85 M_{\odot}$ \\
Distance & $340 \mathrm{pc}$ \\
\hline
\end{tabular}

observed SED, as well as the mid-IR excess including the $10 \mu \mathrm{m}$ silicate emission, is reasonably reproduced, although the model predicts values of the far-IR 60 and $90 \mu \mathrm{m}$ flux well below the observations. However, the model far-IR values could be considered as consistent with the measurements given the broad bandwidth of these far-IR measurements (shown in Figures 5 and 6 as horizontal error bars). This model gives a $\chi_{\text {Total }}^{2}$ value of 1.5 for the combined set of interferometric and SED data. However, if we restrict ourselves to only $2-13 \mu \mathrm{m}$ region of the SED fit, we get a $\chi_{\text {Total }}^{2}(2-13 \mu \mathrm{m})$ value of 1.0. In this model with little or no flaring, which slightly shadows the intermediate distance regions, the outer region of the disk is not efficiently warmed up. Therefore, the $10 \mu \mathrm{m}$ silicate emission is confined in the inner region, which prevents the object from appearing larger at $10 \mu \mathrm{m}$ than at 8 or $13 \mu \mathrm{m}$. The increase in the object size from 8 to $13 \mu \mathrm{m}$ simply results from the emission at longer wavelengths originating from the larger distances from the star.

In order to improve the fit to far-IR SED data further, we also developed a model that flares only in the outer regions, meaning that the inner region is vertically thick enough to shadow a significant part of the disk except the outer regions, resulting in flaring at these regions. The resultant model fit is shown in Figure 6 and the disk parameters are given in Table 5. This model, which is the best-fit model of all those considered, gives a $\chi_{\text {Total }}^{2}$ value of 1.1 for the combined set of interferometric and SED data. Even if we consider only the $2-13 \mu \mathrm{m}$ region of the SED fit, we get a similar value, $\chi_{\text {Total }}^{2}(2-13 \mu \mathrm{m}) \sim 1.1$. The model SED corresponding to the weakly shadowed model compares well with the far-IR SED data, but not the near-IR SED. The errors of the model parameters given in Table 5 refer to the $\chi_{\text {Total }}^{2}+1$ location of the chi-square space. These errors can be underestimated in the case of two or more parameters being degenerate. The scale height of the large grains is found to be about $80 \%$ of that of the small grains, somewhat larger than the value of $60 \%$ used by Tannirkulam et al. (2007). The power-law exponents $p$ and $q$ (Equations (6) and (7)) in the inner regions of the disk are 1.8 and 0.825 , respectively, providing a surface density distribution of $\sum \propto r^{-0.975}$. These parameters for the outer flaring regions are 1.8 and 1.125 , respectively, providing a surface density distribution of $\sum \propto r^{-0.675}$. The corresponding model images at $2.17,3.81,8.21,9.73$, and $12.71 \mu \mathrm{m}$ for this best-fit weakly shadowed disk model are given in Figure 7. These images suggest that the $K$-band data are dominated by the emission from the inner rim, while the extended emission becomes more and more prominent from the $L$ to the $N$ band. The intensity profiles of the disk along the major and minor axes are shown in Figure 8.

The radial distribution of grain temperature and grain mass density along the midplane for our best-fit weakly shadowed disk model is given in Figure 9. The vertical distance of the $\tau_{\lambda}=1$ surface at $2.17,3.81,8.21,9.73$, and $12.71 \mu \mathrm{m}$ of this model is also given in Figure 9. The height of $\tau_{\lambda}=1$ surfaces reflect the wavelength dependence of the opacity. The $\tau_{\lambda}=1$ surface for $3.81 \mu \mathrm{m}$ is higher than that for $2.17 \mu \mathrm{m}$ because the scattering coefficient of the $2 \mu \mathrm{m}$-sized grains shows a peak at 3-4 $\mu \mathrm{m}$. Likewise, the surface for $9.73 \mu \mathrm{m}$ is higher than those for 8.21 and $12.71 \mu \mathrm{m}$ because of the $10 \mu \mathrm{m}$ silicate feature. The vertical distance of $\tau_{\lambda}=1$ surface goes down at large radii. This is because there are two competing factors in determining this surface: (1) density decreases in the radial direction and (2) scale height increases at larger radii. At large radii, the former factor is greater than the latter, leading to the downturn of the

Table 5

Derived Parameters for the Circumstellar Disk

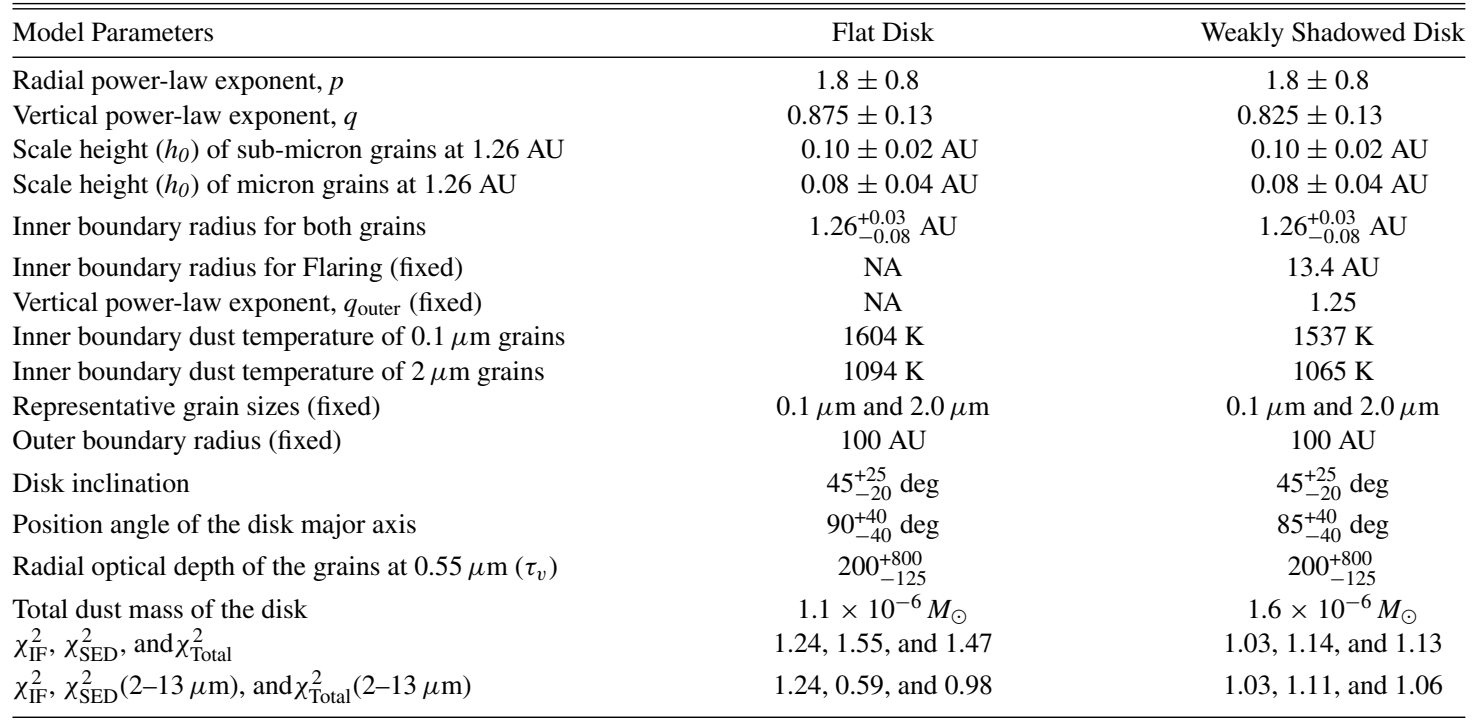

Notes. The entries $\chi_{\mathrm{IF}}^{2}, \chi_{\mathrm{SED}}^{2}$, and $\chi_{\text {Total }}^{2}$ refer to the $\chi_{R}^{2}$ for interferometric data, SED data, and the combined set of interferometric and SED data, respectively. $\chi_{\mathrm{SED}}^{2}(2-13 \mu \mathrm{m})$ and $\chi_{\text {Total }}^{2}(2-13 \mu \mathrm{m})$ refer to the $\chi_{R}^{2}$ for SED data, and the combined set of interferometric and SED data in the $2-13 \mu \mathrm{m}$ region. 

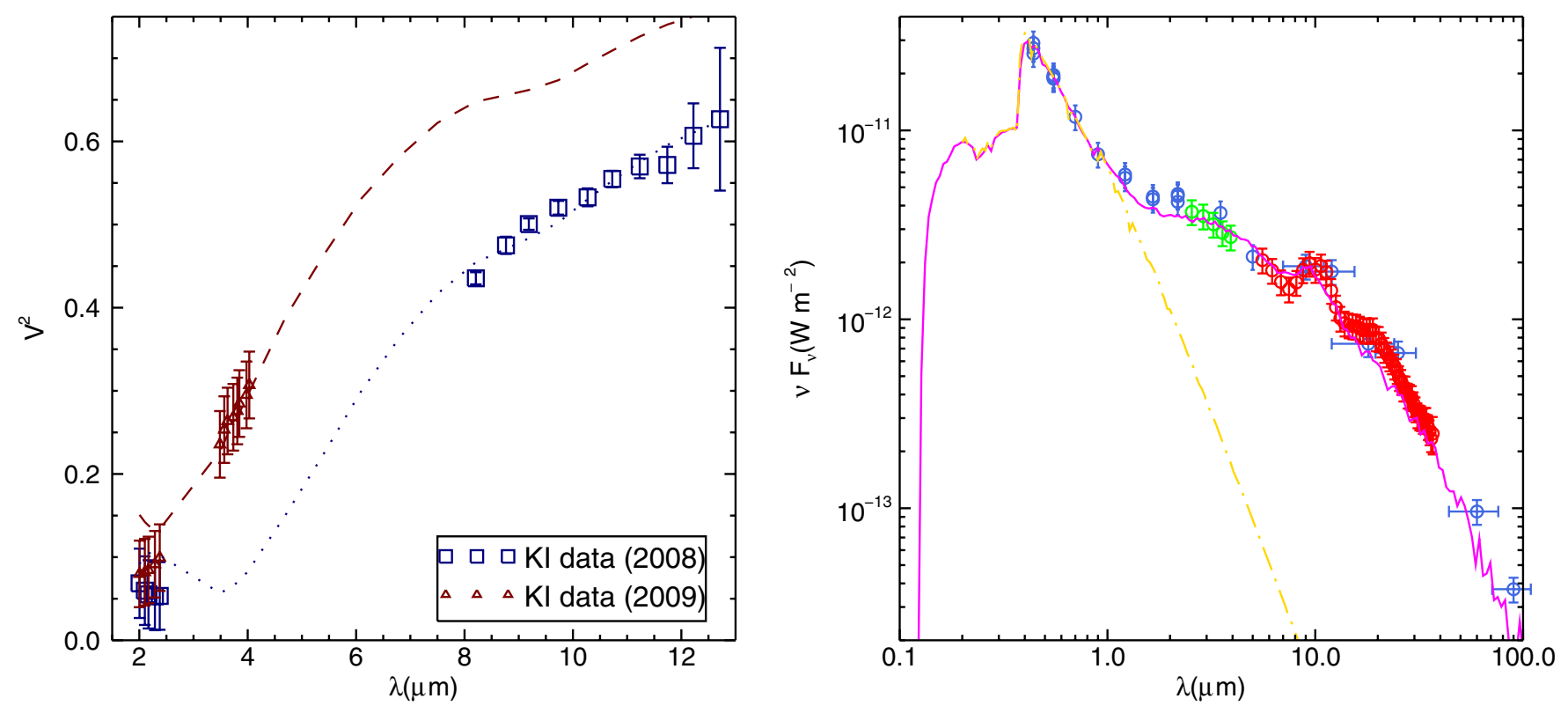

Figure 5. Radiative transfer model fits. Left: multi-wavelength $V^{2}$ measurements and model $V^{2}$ Monte Carlo simulations (see the text for details). The dotted and dashed lines represent visibilities predicted by the 2D radiative transfer model of a flat disk for the baseline orientation of the two epochs shown along with symbols as in Figure 3. Right: the dash-dotted and solid lines are model SEDs of the star and star-plus-disk, respectively, while symbols are the same as in Figure 3. The bandwidth (FWHM) of the IRAS and Akari photometric data are shown as horizontal error bars.
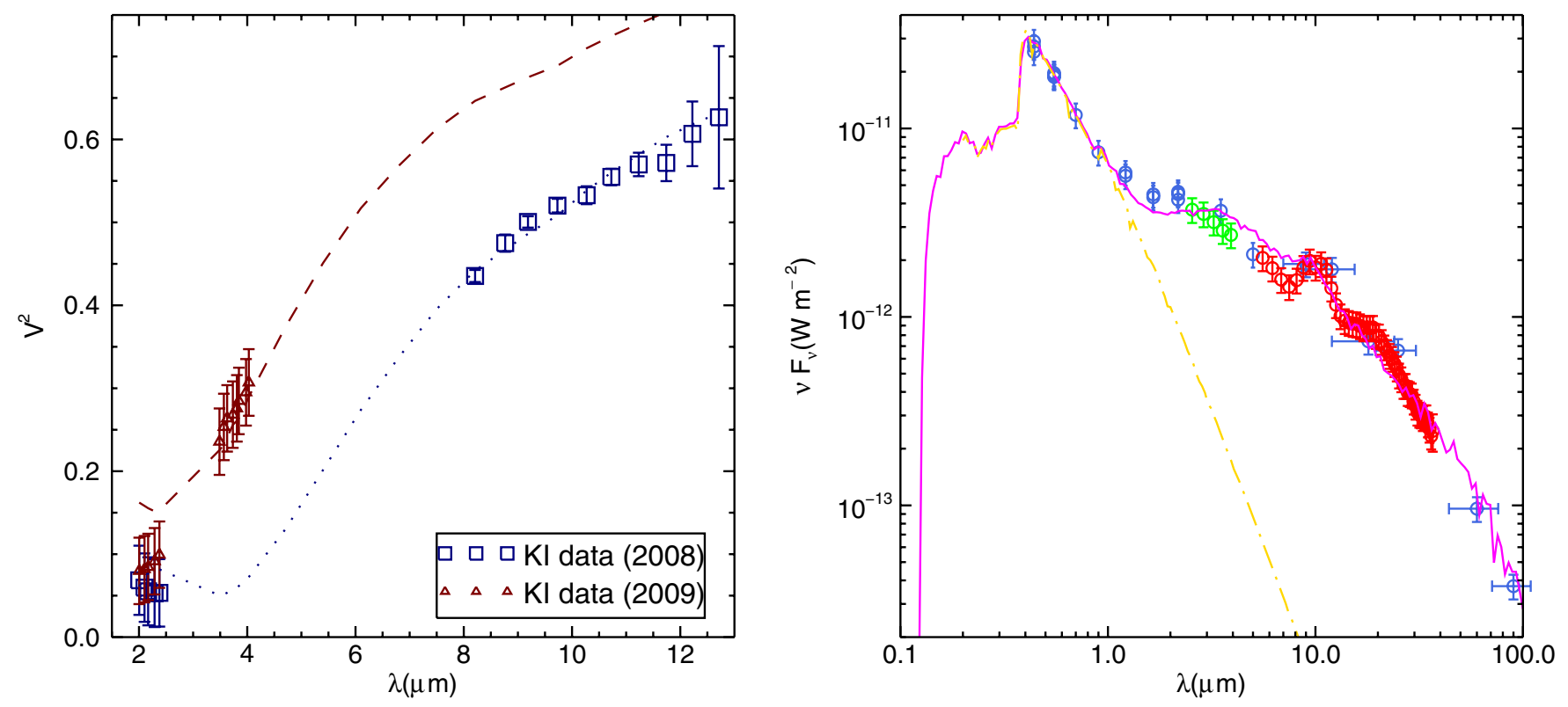

Figure 6. Same as Figure 5 but for a weakly shadowed disk.

$\tau_{\lambda}=1$ surface. Moreover, the plot does not go all the way to the outer radius $(=100 \mathrm{AU})$ because the optical depth measured from the vertical infinity does not reach 1 before the midplane at radii larger than some value, which depends on wavelength. The peak value of the $\tau_{\lambda}=1$ surface is in the range $0.25-0.45 \mathrm{AU}$. As the upper panels of Figure 11 show, the temperatures at these vertical distances are not different from that of the midplane and are more or less same in the $2-13 \mu \mathrm{m}$ region. A representative temperature profile at $3.81 \mu \mathrm{m}$ (where the vertical distance of the $\tau_{\lambda}=1$ surface is maximum) of the best-fit weakly shadowed disk model is shown in Figure 9 (bottom right).

In a flared disk, the stellar and any other high energy flux is absorbed and re-radiated in the upper disk atmosphere layers. Midplane heating is possible either via re-radiation or directly if stellar photons entering from the top of the disk atmosphere can propagate downward to the midplane. However, in the case of MWC 325, flaring becomes important only beyond $13 \mathrm{AU}$ from the star (see Figure 9, upper left panel). While the argument above about the stellar flux possibly heating the midplane of the disk is appropriate for the flared regions, where there is a geometrically large area to intercept radiation from the star, at small disk radii this is not the case. The radius of the star is only $0.017 \mathrm{AU}$ in size compared to the $0.1 \mathrm{AU}$ scale height of the disk at $1.26 \mathrm{AU}$. Thus, the star can only effectively illuminate the inner radius of the disk and it of course illuminates some of the disk, but that material is of low density, being a few scale heights from the midplane, and hence can only weakly heat the midplane. The material has to be something 

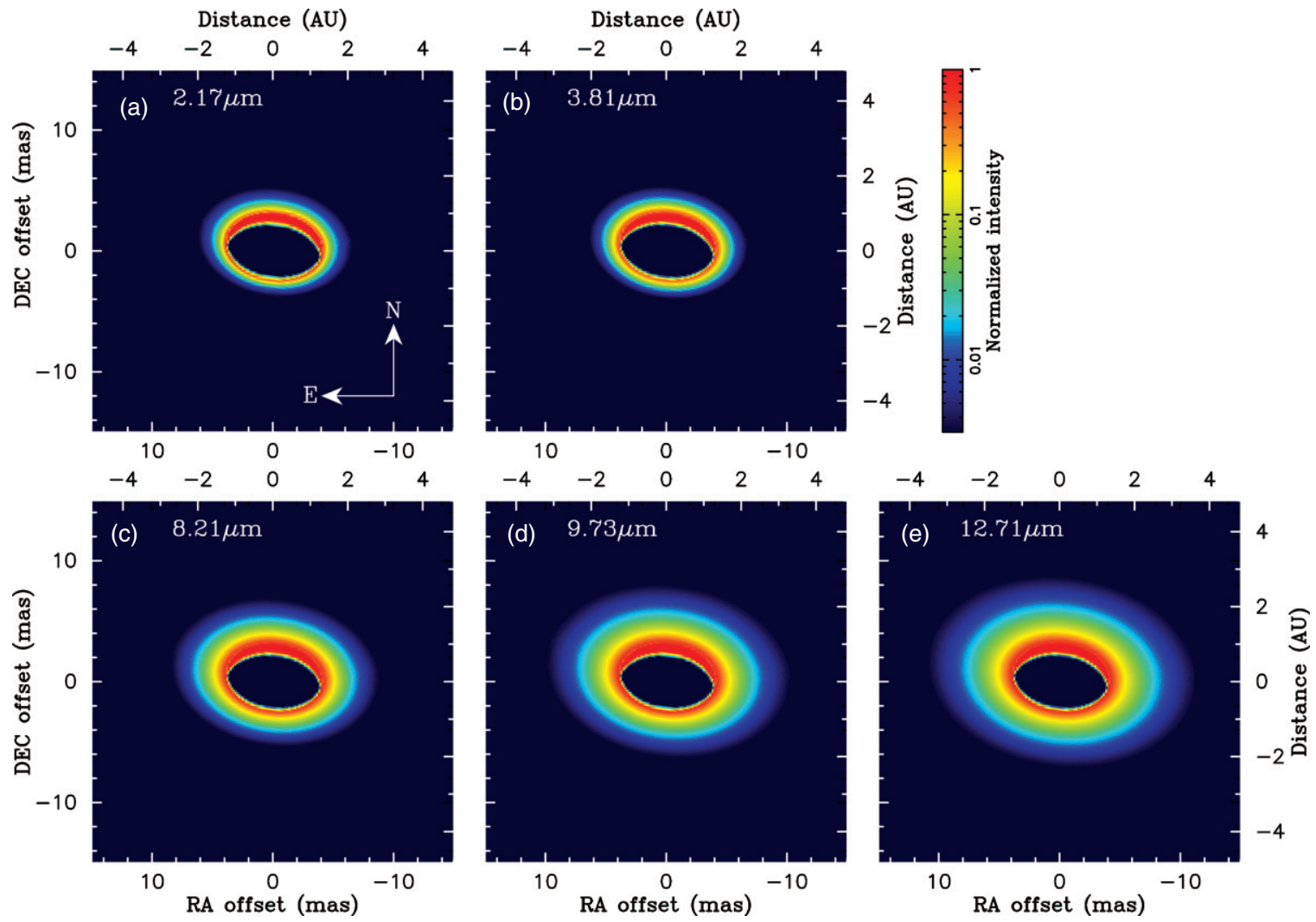

Figure 7. Model images in logarithmic scale. Weakly shadowed disk model images generated through radiative transfer modeling of multi-wavelength interferometric measurements and SED data are shown at 2.17, 3.81, 8.21, 9.73, and $12.71 \mu \mathrm{m}$ (see the text for details). The disk parameters are given in Table 5 (right column).
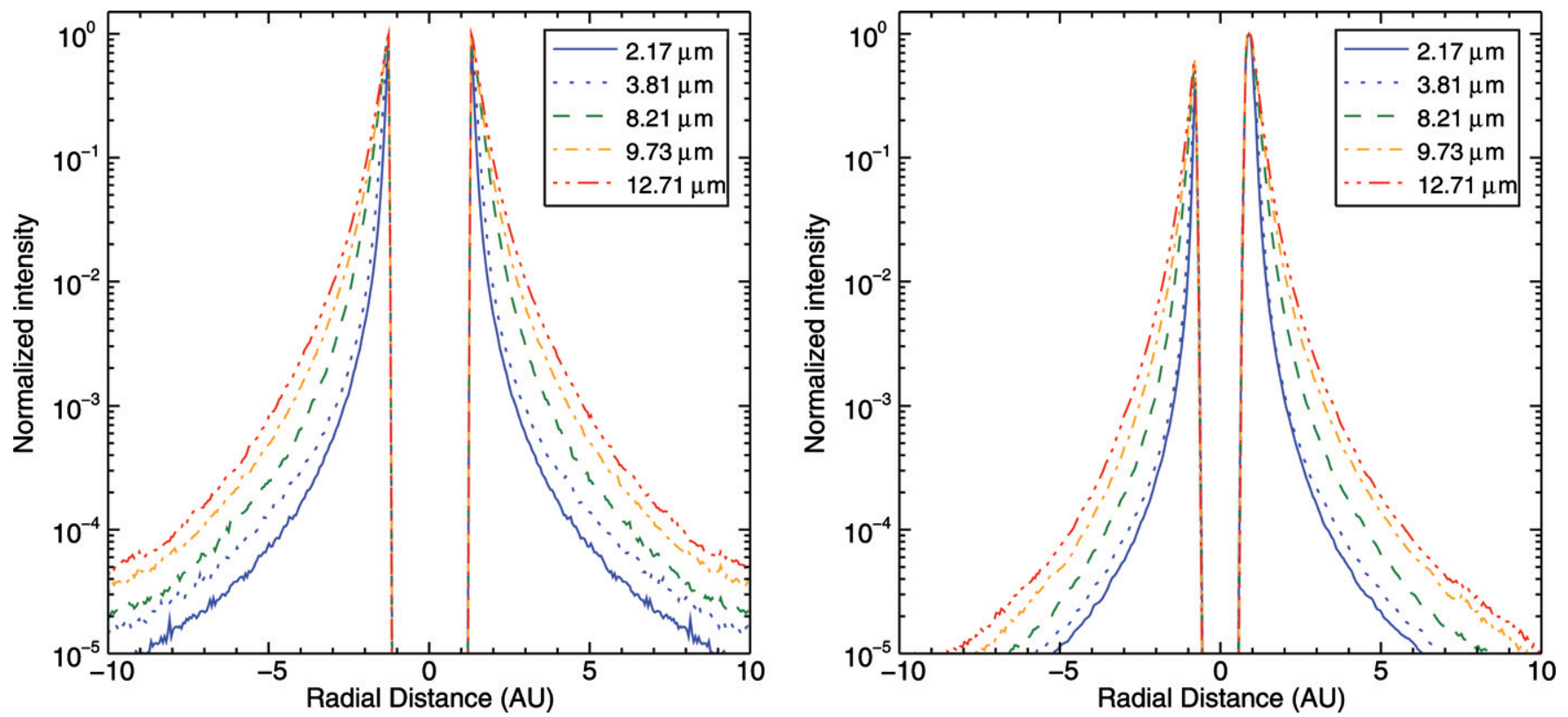

Figure 8. Left: intensity profile of the best-fit weakly shadowed disk model shown in Figure 7 at 2.17, 3.81, 8.21, 9.73, and $12.71 \mu \mathrm{m}$ along the major axis. Right: same as that of the left figure but for the minor axis.

greater than about $4^{\circ}$ (i.e., $\left.\tan ^{-1}((0.1-0.017) / 1.26)\right)$ in angle from the star off the midplane to get mostly pure stellar radiation.

We derive a total dust mass of $9.9 \times 10^{-8} M_{\odot}$ for sub-micron grains and $1.5 \times 10^{-6} M_{\odot}$ for the micron grains, assuming a bulk density of $3.5 \mathrm{gm} \mathrm{cm}^{-3}$ and $2.24 \mathrm{gm} \mathrm{cm}^{-3}$ for the silicate and graphite grains (Zubko et al. 2004) and hence $2.87 \mathrm{gm} \mathrm{cm}^{-3}$ for the silicate-graphite (50:50 ratio) mixture. Thus, the derived total dust-mass of the sub-micron- and micron-sized grains in the disk of MWC 325 is $1.6 \times 10^{-6} M_{\odot}$.

\section{DISCUSSION}

Our new spectrally dispersed $K-, L-$, and $N$-band KI observations provide powerful new constraints on the physical structure of the material surrounding the young Herbig Ae star MWC 325. 

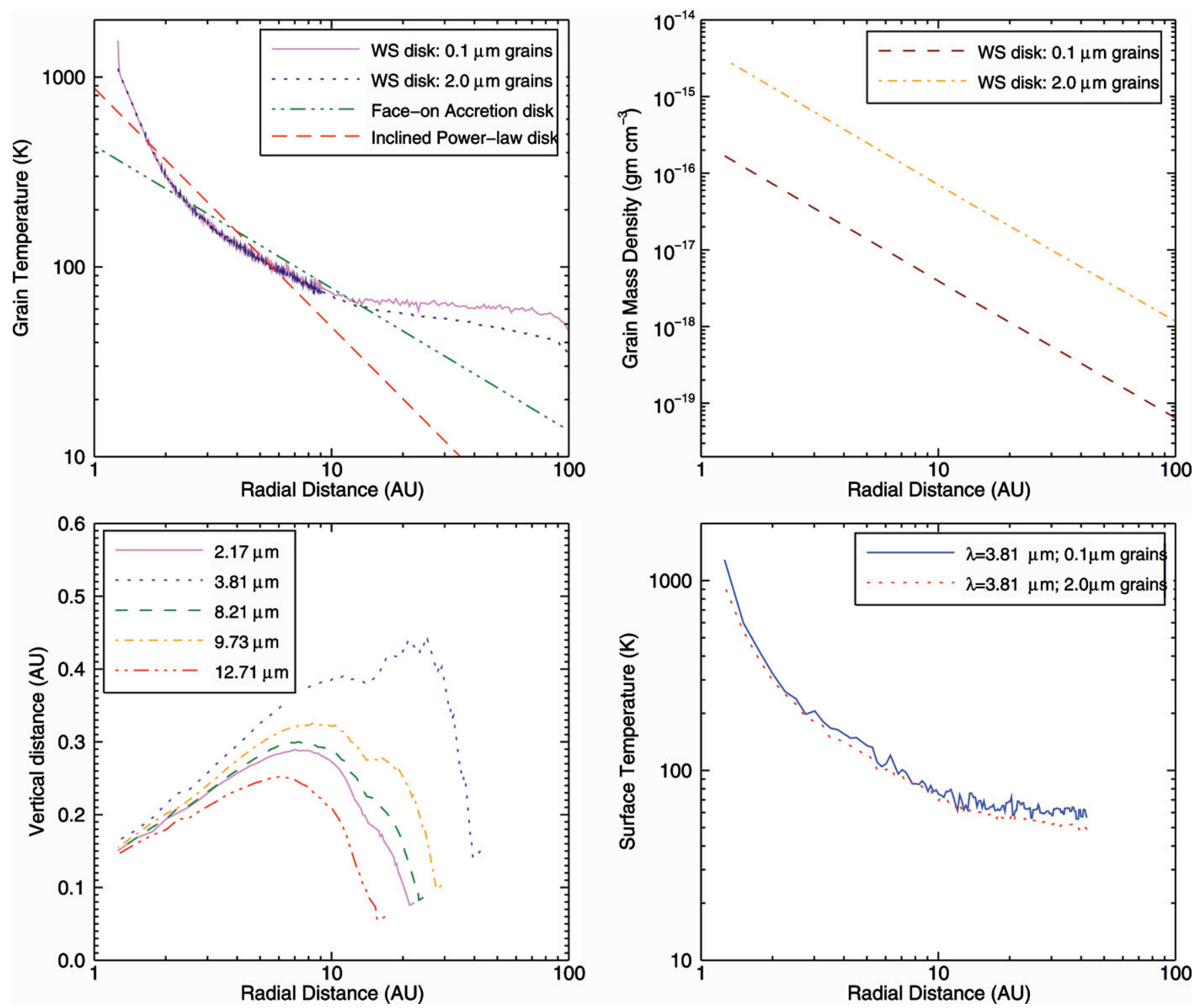

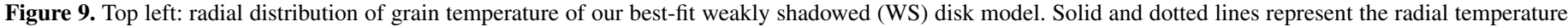

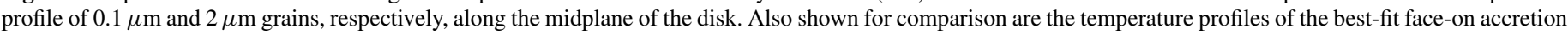

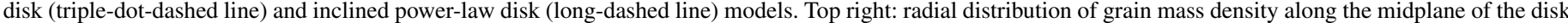

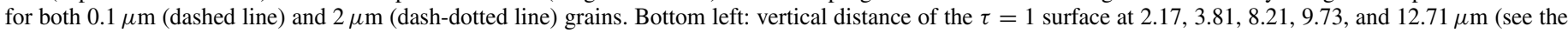

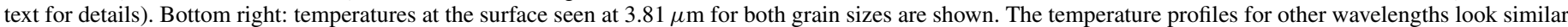
and are not shown here for clarity (see the text for details).

Wavelength-dependent uniform-disk sizes of our measurements have a steep slope (i.e., size increases with wavelength) confirming that the $2-12 \mu \mathrm{m}$ emission region is extended with strong radial temperature dependence.

For MWC 325, the derived uniform-disk diameter at $10 \mu \mathrm{m}$ $\left(\phi_{10 \mu \mathrm{m}}=12.3 \pm 0.2 \mathrm{mas}\right)$ is about a factor of 2.2 larger than the diameter at $2.2 \mu \mathrm{m}\left(\phi_{2.2 \mu \mathrm{m}}=5.7 \pm 0.3 \mathrm{mas}\right)$, and the derived mean uniform-disk diameter at $3.7 \mu \mathrm{m}\left(\phi_{3.7 \mu \mathrm{m}}=7.7 \pm\right.$ 0.2 mas) is about a factor of 1.4 larger than the diameter at $2.2 \mu \mathrm{m}$. The derived $K$-band uniform-disk angular diameter is consistent with the reported $K$-band value of $5.57_{-0.04}^{+0.04}$ mas by Eisner et al. (2004). Monnier et al. (2006) reported a ring diameter of $3.48 \pm 0.4$ mas in the $H$ band. Eisner et al. (2009) used a dust-plus-gas model to fit their spectrally dispersed $(R \sim 230) \mathrm{KI}$ observations in the $K$ band. These authors report a dust ring diameter of $4.28 \pm 0.07$ mas at $1106 \pm 10 \mathrm{~K}$, and an inner gas diameter of $0.97 \pm 0.07$ mas at $3115 \mathrm{~K}$. Our modeling shows that the observed SED and KIN data can be explained without hot emission from inside the dust sublimation radius. This can indeed mean the absence of the hot emission found in some Herbig Be stars (due to hot gas or highly refractory dust). However, since we did not test a model with such hot emission, we cannot make a definite conclusion about this.

The disk inclination angle of $45_{-20}^{+25}$ deg derived from our radiative transfer calculations is consistent with that of the inner rim of $18_{+30}^{-18} \mathrm{deg}$ derived from our elliptical ring model fit to broadband $K$-band measurements within the measurement accuracy. Moreover, the best-fit radiative transfer model also fits $K$-band measurements well $\left(\chi_{\mathrm{IF}}^{2}=1.3\right)$. Earlier, Isella et al. (2006) report disk inclination angle in the range $40^{\circ}-65^{\circ}$ using $H$ - and $K$-band interferometry data available in the literature, which is somewhat larger than our value. And their inner boundary radius of $0.7 \mathrm{AU}$ is about a factor of two smaller than our value of 1.26. While our analysis incorporates sub- 


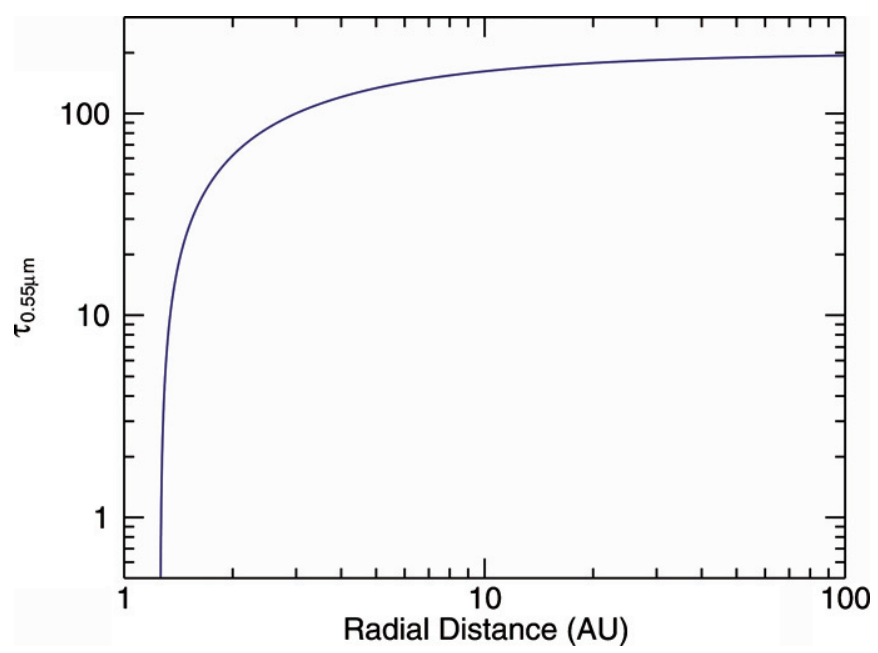

Figure 10. Radial distribution of optical depth along the midplane of the best-fit weakly shadowed disk, i.e., at latitude $0^{\circ}$. As can be seen, the disk is optically thick at the inner regions along the midplane shielding the outer regions from the stellar radiation.

(A color version of this figure is available in the online journal.)

micron $(0.1 \mu \mathrm{m})$ and micron $(2 \mu \mathrm{m})$ radius grains to fit the interferometric and SED data simultaneously, Isella et al. (2006) used $0.3 \mu \mathrm{m}$ and $\geqslant 1.2 \mu \mathrm{m}$ radius grains to fit simultaneously near-infrared interferometric data and optical and near-infrared SED data (far-infrared SED data was not included in their analysis). Kraus et al. (2008b) used similar small $(0.005-1 \mu \mathrm{m})$ and large $(1-1000 \mu \mathrm{m})$ radius grains to simultaneously fit a broad wavelength range of interferometric and SED data of the Herbig Be star MWC 147. We suggest that the grains in the disk of MWC 325 have not yet grown to millimeter-sized grains. Perhaps the luminosity of the central star plays a major role in promoting the grain growth process.

The radial distribution of grain temperature of MWC 325 along the $\tau_{\lambda}=1$ surface of the disk (Figure 9) in the 1-2 AU region is steeper than that of a classic disk with a powerlaw exponent of -0.75 , and the temperature gradient becomes shallower at larger radial distances. For deriving a power-law index of -0.75 , the disk is assumed to be directly irradiated by the star. However, this is not the case for the material in the $\tau_{\lambda}=1$ surface, except at the inner rim. Figure 10 shows the optical depth at $0.55 \mu \mathrm{m}$ in the midplane measured from the inner rim. The figure shows that the optical depth to the region even slightly behind the inner rim is already higher than 1 , and therefore, the $\tau_{\lambda}=1$ surface of the disk beyond the inner-rim region is shielded from the stellar radiation, resulting in reduced grain temperature (see the temperatures around the midplane at three representative radial positions in Figure 11) and hence a steeper temperature gradient.

Earlier multi-band interferometry has shown a somewhat similar temperature profile for two Herbig Ae disks, namely, MWC 275 and AB Aur (Tannirkulam et al. 2008a), and to a lesser extent of agreement for the Herbig Be disk MWC 147 (Kraus et al. 2008b). Recent disk models include an inner rim at the boundary between the dust-free inner disk and the main disk that is vertically "puffed up" relative to the dusty material at only slightly larger radii. Modifications to this simple geometry and with some hot refractory dust or gaseous material inside the inner rim and a blunt (rather than sharp) dust boundary can have just the effect we see of an apparent size gradient with wavelength and an accompanying steep temperature profile (see, e.g., the recent review by Dullemond \& Monnier 2010). Radial sorting of grain sizes may also play a role in the effects that we see.

We assumed a grain composition consisting of a mixture of graphite and silicate with equal fractional abundances for the radiative transfer models presented in this paper. We also attempted a grain composition of Silicate and amorphous carbon with equal abundances. We find that such a grain composition generates a weak $10 \mu \mathrm{m}$ feature. The reason is that graphite has steeper wavelength dependence $\left(\sim \lambda^{-2}\right)$ than amorphous carbon $\left(\sim \lambda^{-1.2}\right)$. Because of the flatter wavelength dependence, amorphous carbon has a higher opacity at $10 \mu \mathrm{m}$ than graphite, which makes the silicate feature less pronounced. In order to improve the strength of this feature (to be consistent with SED data), we had to introduce significant flaring in the disk as this would expose more and more disk material to stellar radiation, which produces the pronounced silicate feature. However, such flared-disk geometry fails to reproduce the shape of the observed visibility spectrum. The corresponding best-fit model gives an unacceptably large $\chi^{2}$ value $\left(\chi_{\mathrm{IF}}^{2} \sim 56\right)$ for our interferometric data although the fit to SED data is reasonable $\left(\chi_{\mathrm{SED}}^{2} \sim 2\right)$. Hence, we ruled out silicate-amorphous carbon grain composition for the disk of MWC 325. A silicate-only model simultaneously fits the visibility and SED data. However, such grain composition is far from that of the interstellar grains. Therefore, we did not consider this case in our work.

The decomposition of the $N$-band silicate feature of MWC 325 shows a strong dominance of $2 \mu \mathrm{m}$ amorphous silicate grains with a mass fraction of 0.85 (Schütz et al. 2009). Sub-micron $(0.1 \mu \mathrm{m})$ amorphous silicate and enstatite and $2 \mu \mathrm{m}$ silica grains are present in much smaller amounts. More recently, Juhász et al. (2010) decomposed mid-infrared spectra (5.5-37 $\mu \mathrm{m})$ of a sample of Herbig Ae/Be stars taken with the Spitzer Space Telescope. They used a mixture of five-grain species, namely, amorphous silicate with olivine and pyroxene stoichiometry, crystalline forsterite, and enstatite, and silica, together with PAHs, and report a mass fraction of 0.68 for micronsized amorphous silicate grains. In our best model, the mass fraction of the $2 \mu \mathrm{m}$ grains is 0.94 . Thus, our model is qualitatively consistent with Juhász et al. (2010) and Schütz et al. (2009), who both conclude that micron-sized amorphous silicate grains dominate in MWC 325. Direct comparison of our grain composition with these mid-infrared modeling papers is not possible since the mid-infrared decomposition method is sensitive only to dust grains from the surface of the disk that show resonances in the mid-infrared (silicate-based grains). Thus featureless grains such as amorphous carbon or iron are unaccounted for here. High spectral resolution interferometric measurements in the mid-infrared are essential to study the presence of crystalline grains since they produce narrow features.

Earlier studies (Monnier \& Millan-Gabet 2002; Eisner et al. 2004) using broadband, usually single-wavelength, interferometric data, recognized a difference in the near-infrared size vs. luminosity behavior of high luminosity objects (pre-mainsequence $\mathrm{Be}$ ) compared to lower luminosity ones (pre-mainsequence $\mathrm{Ae}$ ), the former being more consistent with "classical disk" models. This has been revisited most recently by Vinković \& Jurkić (2007), who use a model-independent comparison of visibility to scaled baseline and find a distinction between

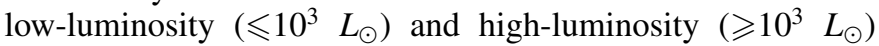
YSO disks where the luminosity break point corresponds to an approximate spectral type of B3-B5. Those authors modeled 

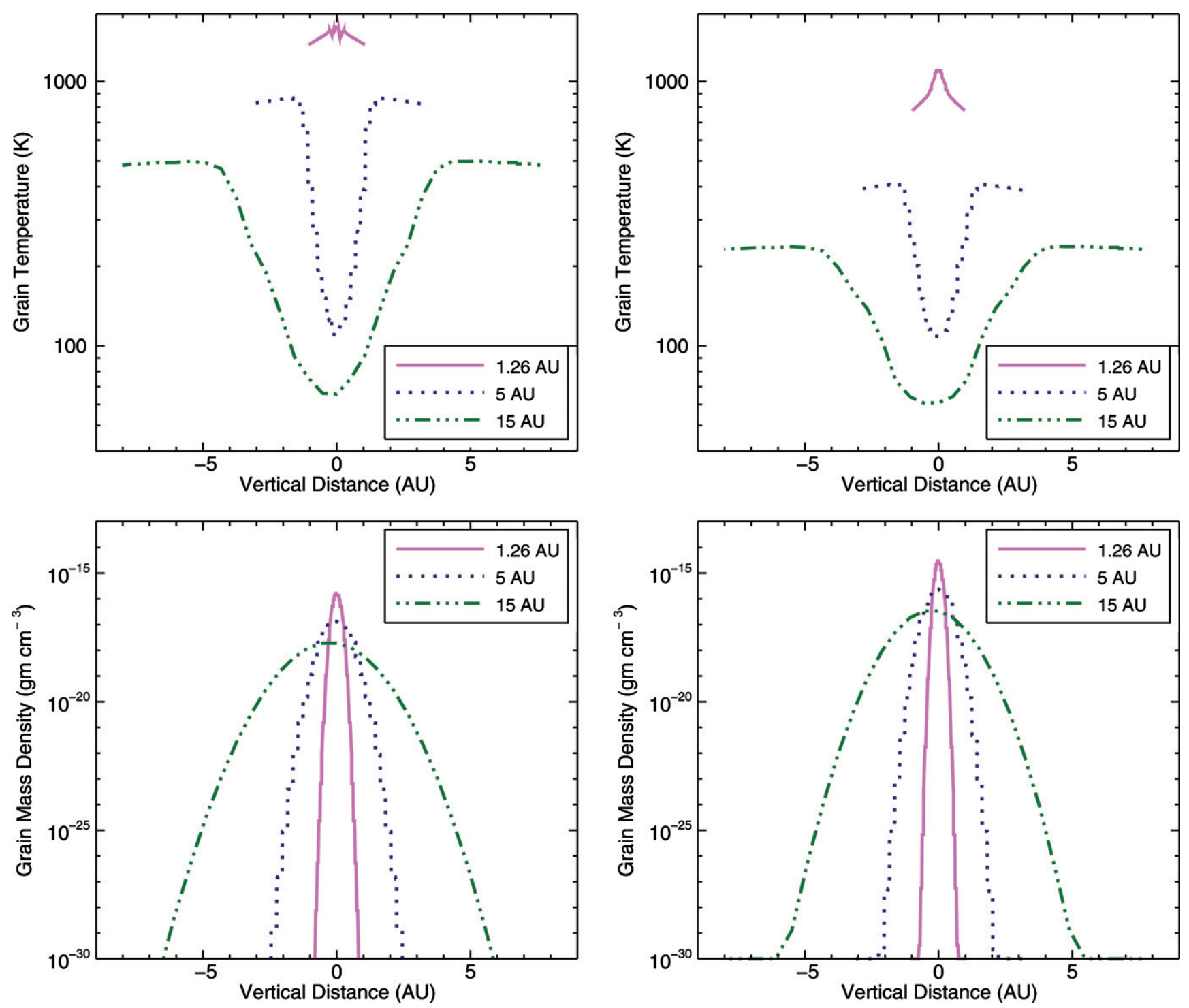

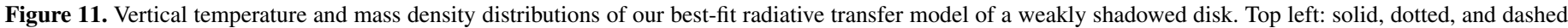

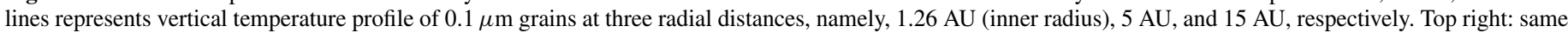

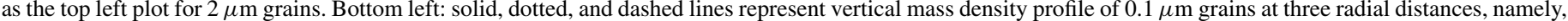
1.26 $\mathrm{AU}$ (inner radius), $5 \mathrm{AU}$, and $15 \mathrm{AU}$, respectively. Bottom right: same as the bottom left plot for $2 \mu \mathrm{m}$ grains.

the visibility clusters of low luminosity Herbig Ae/Be stars with optically thick rings of $0^{\circ}-60^{\circ}$ inclination at a dust sublimation temperature of $\sim 1500 \mathrm{~K}$. The alternate model used to fit these visibility clusters was a dusty halo model with optical depths of $\sim 0.15-0.8$. They modeled visibility clusters of high luminosity Herbig Be stars with a classical accretion disk model and $\mathrm{T}$ Tauris stars with a dusty halo model.

However, multi-wavelength interferometric studies have not always supported these conclusions when objects are modeled in detail (Kraus et al. 2008b; Acke et al. 2008; Ragland et al. 2009). Our earlier multi-wavelength results (Ragland et al. 2009) show that MWC 419 (B8, $\left.330 L_{\odot}\right)$ has disk characteristics of a highluminosity object in the categories of Monnier \& Millan-Gabet (2002). However, it has a luminosity below the break point of $10^{3} L_{\odot}$ identified by Vinković \& Jurkić (2007) and fits within the population of lower luminosity Herbig starts in their modelindependent comparison. Their physical interpretation of the low-luminosity Herbig group is an optically thick disk with an optically thin dust sublimation cavity and an optically thin dusty outflow.
MWC $325\left(\mathrm{~A} 2,83 L_{\odot}\right)$ falls into the low-luminosity group in the classification of Vinković \& Jurkić (2007). Our results presented in this paper do not support their conclusion of a ring or halo model for this low luminosity YSO disk. While a ring model could reproduce, to first order, the bright inner ring region seen from the $K$-band measurements, it would have difficulties explaining the extended disk structures seen from the $L$-band and $N$-band measurements. The apparent discrepancies between our results and that of Vinković \& Jurkić (2007) could be attributed to the fact that their conclusions are based on near-infrared interferometric data while this work incorporated a broader spectral region.

MWC 325 is in the Group II category of Herbig AeBe stars (Acke et al. 2009), in the classification scheme of Meeus et al. (2001), with a relatively weak IR excess and PAH emission compared to the Group I category. These studies based on Spitzer measurements suggest that the disk of MWC 325 is a self-shadowed disk-with an inner rim that is blocking star light from reaching the outer regions of the disk. Our finding of disk geometry with little or no flaring is consistent their results. 


\section{SUMMARY}

This paper reports the first milliarcsecond angular resolution $N$-band nulling and $L$-band $V^{2}$ observations of a Herbig Ae star (MWC 325), along with $K$-band $V^{2}$ data, all spectrally dispersed. This multi-wavelength observational capability is well suited to probing the temperature distribution in the inner regions of YSO disks, which is very important for distinguishing different models and gaining insight into the three-dimensional geometry of the inner disk (see also Ragland et al. 2009).

A simple pole-on uniform disk model was used to infer an increase in size from 2 to $12 \mu \mathrm{m}$, confirming that the disk is extended with a radial temperature gradient. Notably, there is no difference in the wavelength trend of the visibilities within the broad $10 \mu \mathrm{m}$ silicate feature compared to the adjacent continuum. We find that the classical accretion disk and the power-law temperature gradient models fail to fit simultaneously both interferometric and SED data. A two-dimensional slightly shadowed-disk radiative transfer model fits the spectrally dispersed interferometric measurements and the SED data reasonably well. This model implies that the disk surrounding MWC 325 is a nearly flat disk with at most only slight flaring in the outer regions of the disk in contrast to other intermediatemass Ae disks such as MWC 275 and AB Aur, and to their more massive counterparts, such as MWC 419, which exhibit a flat geometry. The dominance of sub-micron grains and the absence of significant flaring in the disk of MWC 325 found from our study suggest that dust-grain growth and dust sedimentation has occurred in the disk of MWC 325. A more complete sample of YSO disk observations with adequate wavelength and $(u, v)$ coverage, plus detailed radiative transfer modeling, are required to address the intriguing inner disk geometry of these sources and to address the structural differences between Herbig Ae and Be disks.

The Keck Interferometer is funded by the National Aeronautics and Space Administration (NASA). Observations presented were obtained at the W. M. Keck Observatory, which is operated as a scientific partnership among the California Institute of Technology, the University of California, and NASA. The Observatory was made possible by the generous financial support of the W. M. Keck Foundation. KI observations were taken through Keck Director's time. We thank E. Appleby, A. Cooper, C. Felizardo, J. Herstein, D. Medeiros, D. Morrison, T. Panteleeva, B. Parvin, B. Smith, K. Summers, K. Tsubota, C. Tyau, E. Wetherell, P. Wizinowich, and J. Woillez for their contributions to KI operations. CHARA observations were taken through NOAO TAC time. We thank Gail Schaefer, P. J. Goldfinger, Chris Farrington, and Theo ten Brummelaar for support of the CHARA observing program and Tabetha Boyajian for advice on data reductions. S.T.R. acknowledges partial support from NASA grant NNH09AK731.

\section{REFERENCES}

Acke, B., Min, M., van den Ancker, M. E., et al. 2009, A\&A, 502, 17

Acke, B., \& van den Ancker, M. E. 2006, A\&A, 457, 171
Acke, B., Verhoelst, T., van den Ancker, M. E., et al. 2008, A\&A, 485, 209

Acke, B., \& Waelkens, C. 2004, A\&A, 427, 1009

Benisty, M., Malbet, F., Dougado, C., et al. 2010c, A\&A, 517, L3

Benisty, M., Natta, A., Isella, A., et al. 2010a, A\&A, 511, 74

Benisty, M., Renard, S., Natta, A., et al. 2011, A\&A, 531, A84

Benisty, M., Tatulli, E., Ménard, F., \& Swain, M. R. 2010b, A\&A, 511, 75

Bjorkman, J. E., \& Wood, K. 2001, ApJ, 554, 615

Cardelli, J. A., Clayton, G. C., \& Mathis, J. S. 1989, ApJ, 345, 245

Catala, C., Alecian, E., Donati, J.-F., et al. 2007, A\&A, 462, 293

Colavita, M. M., Serabyn, E., Booth, A. J., et al. 2008, Proc. SPIE, 7013, 70130A

Colavita, M. M., Serabyn, E., Millan-Gabet, R., et al. 2009, PASP, 121, 1120

Di Folco, E., Dutrey, A., Chesneau, O., et al. 2009, A\&A, 500, 1065

Draine, B. T., \& Lee, H. M. 1984, ApJ, 285, 89

Dullemond, C.P., \& Monnier, J. D. 2010, ARA\&A, 48, 205

Eisner, J. A., Chiang, E. I., Lane, B. F., \& Akeson, R. L. 2007, ApJ, 657, 347

Eisner, J. A., Graham, J. R., Akeson, R. L., \& Najita, J. 2009, ApJ, 692, 309

Eisner, J. A., Lane, B. F., Akeson, R. L., Hillenbrand, L. A., \& Sargent, A. I. 2003, ApJ, 588, 360

Eisner, J. A., Lane, B. F., Hillenbrand, L. A., Akeson, R. L., \& Sargent, A. I. 2004, ApJ, 613, 1049

Eisner, J. A., Monnier, J. D., Woillez, J., et al. 2010, ApJ, 718, 774

Herbig, G. H. 1960, ApJS, 4, 337

Hernández, J., Calvet, N., Hartmann, L., et al. 2005, AJ, 129, 856

Hillenbrand, L. A., Strom, S. E., Vrba, F. J., \& Keene, J. 1992, ApJ, 397, 613

Isella, A., Tatulli, E., Natta, A., \& Testi, L. 2008, A\&A, 483, L13

Isella, A., Testi, L., \& Natta, A. 2006, A\&A, 451, 951

Juhász, A., Bouwman, J., Henning, Th., et al. 2010, ApJ, 721, 431

Koresko, C., Colavita, M., Serabyn, E., Booth, A., \& Garcia, J. 2006, Proc. SPIE, 6268, 626816

Kraus, S., Hofmann, K.-H., Benisty, M., et al. 2008a, A\&A, 489, 1157

Kraus, S., Hofmann, K.-H., Malbet, F., et al. 2009, A\&A, 508, 787

Kraus, S., Preibisch, T., \& Ohnaka, K. 2008b, ApJ, 676, 490

Kurucz, R. L. 1970, SAO Special Report, \#309

Leinert, C., van Boekel, R., Waters, L. B. F. M., et al. 2004, A\&A, 423, 537

Malbet, F., Benisty, M., de Wit, W.-J., et al. 2007, A\&A, 464, 43

Meeus, G., Waters, L. B. F. M., Bouwman, J., et al. 2001, A\&A, 365, 476

Millan-Gabet, R., Schloerb, F. P., \& Traub, W. A. 2001, ApJ, 546, 358

Millan-Gabet, R., Schloerb, F. P., Traub, W. A., et al. 1999, ApJ, 513, L131

Monnier, J. D., Berger, J.-P., Millan-Gabet, R., et al. 2006, ApJ, 647, 444

Monnier, J.D., \& Millan-Gabet, R. 2002, ApJ, 579, 698

Monnier, J. D., Millan-Gabet, R., Billmeier, R., et al. 2005, ApJ, 624, 832

Montesinos, B., Eiroa, C., Mora, A., \& Merín, B. 2009, A\&A, 495, 901

Mora, A., Merin, B., Solano, E., et al. 2001, A\&A, 378, 116

Ohnaka, K., Driebe, T., Hofmann, K.-H., et al. 2006, A\&A, 445, 1015

Pogodin, M. A., Franco, G. A. P., \& Lopes, D. F. 2005, A\&A, 438, 239

Preibisch, Th., Kraus, S., Driebe, Th., et al. 2006, A\&A, 458, 235

Ragland, S., Akeson, R. L., Armandroff, T., et al. 2009, ApJ, 703, 22

Ragland, S., Wizinowich, P., Akeson, R., et al. 2008, Proc. SPIE, 7013, 70130B

Renard, S., Malbet, F., Benisty, M., et al. 2010, A\&A, 519, A26

Schütz, O., Meeus, G., Sterzik, M. F., \& Peeters, E 2009, A\&A, 507, 261

Skrutskie, M. F., Cutri, R. M., Stiening, R., et al. 2006, AJ, 131, 1163

Tannirkulam, A., Harries, T. J., \& Monnier, J. D. 2007, ApJ, 661, 374

Tannirkulam, A., Monnier, J. D., Harries, T. J., et al. 2008a, ApJ, 689, 513

Tannirkulam, A., Monnier, J. D., Millan-Gabet, R., et al. 2008b, ApJ, 677, L51

Tassoul, J.-L. 2000, Stellar Rotation (Cambridge Astrophysics Series 36; Cambridge: Cambridge Univ. Press)

Tatulli, E., Benisty, M., Ménard, F., et al. 2011, A\&A, 531, A1

Tatulli, E., Isella, A., Natta, A., et al. 2007, A\&A, 464, 55

Tuthill, P. G., Monnier, J. D., Danchi, W. C., et al. 2002, ApJ, 577, 826

van Belle, G. T. 1999, PASP, 111, 1515

van den Ancker, M.E., de Winter, D., \& Tjin A Djie, H. R. E. 1998, A\&A, 330, 145

van Leeuwen 2007, A\&A, 474, 653

Vandenbussche, B., Beintema, D., de Graauw, T., et al. 2002, A\&A, 390, 1033

Verhoeff, A.P., Min, M., Acke, B., et al. 2010, A\&A, 516, A48

Vinković, D., \& Jurkić, T. 2007, ApJ, 658, 462

Weigelt, G., Grinin, V.P., Groh, J.H., et al. 2011, A\&A, 527, A103

Zubko, V., Dwek, E., \& Arendt, R.G. 2004, ApJS, 152, 211 